\title{
The Supershell-Molecular Cloud Connection: Large-Scale Stellar Feedback and the Formation of the Molecular ISM
}

\author{
J. R. Dawson ${ }^{1,2}$ \\ ${ }^{1}$ School of Mathematics and Physics, University of Tasmania, Sandy Bay Campus, Churchill Avenue, Sandy Bay, TAS 7005, Australia \\ ${ }^{2}$ Email: joanne.dawson@utas.edu.au
}

(Received December 5, 2012; AccePted January 7, 2013; Online Publication February 28, 2013)

\begin{abstract}
The accumulation, compression, and cooling of the ambient interstellar medium (ISM) in large-scale flows powered by OB cluster feedback can drive the production of dense molecular clouds. We review the current state of the field, with a strong focus on the explicit modelling and observation of the neutral ISM. Magnetohydrodynamic simulations of colliding ISM flows provide a strong theoretical framework in which to view feedback-driven cloud formation, as do models of the gravitational fragmentation of expanding shells. Rapid theoretical developments are accompanied by a growing body of observational work that provides good evidence for the formation of molecular gas via stellar feedback-both in the Milky Way and the Large Magellanic Cloud. The importance of stellar feedback compared with other major astrophysical drivers of dense gas formation remains to be investigated further, and will be an important target for future work.
\end{abstract}

Keywords: galaxies: ISM, ISM: bubbles, ISM: clouds, ISM: evolution, ISM: structure, stars: formation

\section{INTRODUCTION}

The formation of dense, star-forming gas from the diffuse interstellar medium (ISM) is a key process in the evolution of galaxies, and a major unsolved problem in astrophysics. While the accumulation, cooling, and fragmentation of the ISM into self-gravitating, star-forming structures has been extensively studied-particularly from a theoretical perspective- the subject remains a challenging one, involving the interplay between a complex array of physical, chemical, and astrophysical processes. Many key questions remain unanswered, including which astrophysical drivers are responsible for the majority of dense gas formation, the timescales of cloud formation and destruction, and how the details of the fragmentation process set the basic foundations for subsequent star formation. In the present-day, metal-rich universe, the ISM in star-forming clouds is almost always in the molecular phase. The key question can therefore be phrased as: How, when, and where do we form molecular clouds?

The basic ingredients for molecular cloud formation are high densities and high column densities. The former is essential for effective cooling (and also facilitates rapid molecule formation) and the latter is necessary to shield the gas from UV heating (and also prevents the photodissociation of molecular species). Molecular cloud formation there- fore typically involves large-scale compressive events capable of piling large quantities of material into small volumes. Astrophysical drivers include global gravitational instabilities in galaxy discs (e.g. Wada, Spaans, \& Kim 2000; Kim, Ostriker, \& Stone 2002; Tasker \& Tan 2009; Bournaud et al. 2010), the accumulation of matter in spiral shocks (e.g. Kim \& Ostriker 2006; Dobbs, Bonnell, \& Pringle 2006; Dobbs \& Bonnell 2008), and compression in expanding shells driven by stellar feedback (e.g. McCray \& Kafatos 1987; Hartmann, Ballesteros-Paredes, \& Bergin 2001; Ntormousi et al. 2011), all aided by turbulence that acts to enhance density on a range of scales (e.g. Elmegreen 2002; Mac Low \& Klessen 2004; Glover \& Mac Low 2007; McKee \& Ostriker 2007; Federrath \& Klessen 2012).

This review will examine the role of large-scale stellar feedback in the formation and evolution of molecular clouds. This route to molecular cloud formation is of particular interest, since it is the key element of how star formation self-regulates. The cumulative energy input from multiple stellar winds and supernovae may form new molecular gas through the sweep up and sustained compression of the ambient atomic medium in giant 'supershells' around OB clusters. Rapid cooling of this swept-up gas-in combination with gravitational, fluid dynamical, and thermal instabilitiesresults in the fragmentation of supershell walls into dense clouds, which will become molecular (and eventually star 
forming) where density and column density requirements are met. This process is often discussed in the context of the flow-driven model of molecular cloud formation, which envisions dense gas and star formation as a rapid, dynamical process, and molecular clouds as short-lived entities that form at the interfaces of turbulent flows (see Walder \& Folini 1996; Hennebelle \& Pérault 1999; Elmegreen 2000; Hartmann et al. 2001; Audit \& Hennebelle 2005; Vázquez-Semadeni et al. 2006, 2007; Hennebelle et al. 2008; Heitsch \& Hartmann 2008; Banerjee et al. 2009; Inoue \& Inutsuka 2012; Clark et al. 2012). Magnetohydrodynamical models of this process have been developing rapidly in the last decade.

Despite theoretical advances, the role of large-scale stellar feedback in molecular cloud formation remains an outstanding question. It is clear that the feedback from massive stars plays a key role in both structuring and supporting the disc ISM (e.g. de Avillez \& Berry 2001; Joung, Mac Low, \& Bryan 2009; Dobbs, Burkert, \& Pringle 2011; Hill et al. 2012; Hopkins, Quataert, \& Murray 2012), and is likely responsible for triggering a significant fraction of observed star formation on local scales in existing molecular clouds (e.g. Boss 1995; Yamaguchi et al. 1999b; Hosokawa \& Inutsuka 2006; Dale, Bonnell, \& Whitworth 2007; Leão et al. 2009; Deharveng et al. 2010). However, its role in the initial production of dense gas is not well constrained. There is evidence to suggest that the formation of dense clouds and clumps on galactic scales is primarily driven by a combination of global gravitational instability and turbulence (e.g. Wada \& Norman 2001; Elmegreen 2002; Tasker \& Tan 2009; Bournaud et al. 2010; Mac Low \& Glover 2012), with stellar feedback relegated to a secondary role. In this picture, global self-gravity produces large dense gas complexes and spiral arms, while the localised energy input from OB clusters both triggers star formation in existing clouds and drives the formation of some extra molecular material (Elmegreen 2002). The magnitude of the stellar feedback contribution, however, remains to be confirmed.

Observationally, there is a large body of literature that makes at least some reference to the formation, evolution, and destruction of molecular clouds in relation to feedback supershells or superbubbles (e.g. Jung, Koo, \& Kang 1996; Patel et al. 1998; Fukui et al. 1999; Kim \& Koo 2000; Matsunaga et al. 2001; Yamaguchi et al. 2001a,b; Dawson et al. 2008b, 2011a, 2011b, 2013). However, much of this work consists of case studies of individual objects, in which it is difficult to conclusively demonstrate triggered cloud formation, and discussion on the origin of the molecular gas is often speculatory. One challenge is that the real ISM is structurally very complex. It is characterised by a frothy structure of loops, filaments, carved out tunnels, and irregular interlocking shells and bubbles, exhibits fractal structure across all size scales, and is peppered with molecular clouds whose individual evolutionary histories are ambiguous. The interaction of supershell shock fronts with pre-existing dense gas is also common, and further complicates the interpretation of associated clouds. Until recently, the limitations of molecular line observations meant that most work on the molecular ISM in supershells was carried out in the Milky Way, where lineof-sight confusion exacerbates observational difficulties. It has therefore proved challenging to address the role played by large-scale stellar feedback to molecular cloud in a quantitative sense or examine its importance in entire galactic systems. Nevertheless, when taken as a whole, the literature provides a good case for molecular cloud formation in supershell walls, and there is now a growing body of work approaching the problem from a more quantitative or statistical standpoint, spurred on by ever-improving observational capabilities (e.g. Matsunaga et al. 2001; Yamaguchi et al. 2001b; Book et al. 2009; Dawson et al. 2008b, 2011b).

This paper reviews the theoretical basis and observational evidence for feedback-driven molecular cloud formation, focussing primarily on the evolution of the neutral ISM and the relationship between supershells and the molecular medium. In Section 2, we provide some background on the feedbackstructured ISM, the evolution of a classical shell, and a brief overview of the ways in which supershells may influence the molecular ISM. Section 3 deals with the theory and modelling of molecular cloud formation, including the gravitational fragmentation of expanding shells, the colliding flows model of molecular cloud formation, and numerical simulations of the ISM in galaxy discs. In Section 4, we touch briefly on the relevant theory pertaining to the interaction of a supershell shock front with existing dense clouds, although the complex subject of triggered star formation is given only a brief mention. Section 5 describes observations of molecular gas in feedback superstructures, working outwards from the local ISM, to the Milky Way as a whole, to the Magellanic Clouds, and presenting strong evidence that molecular cloud formation via large-scale stellar feedback is occurring both in the Galaxy and in the Large Magellanic Cloud (LMC). Finally, Section 6 provides a brief summary and outlines some directions for future development.

\section{BACKGROUND}

\subsection{The galactic bubble bath}

The energy input from massive stars significantly impacts the structure and evolution of the ISM. The ISM of star-forming galaxies is riddled with the footprints of this stellar feedback, in the form of cool shells, hot bubbles and evacuated cavities seen in multiple tracers, from X-ray to radio. The existence of such structures in the ISM has been extensively documented throughout the last century (see review by Tenorio-Tagle \& Bodenheimer 1988), and led Brand \& Zealey (1975) to coin the phrase 'the cosmic bubble bath' to describe the structure of the Galactic disc. The volume-filling factor of feedback bubbles in the Milky Way is thought to lie somewhere between $\sim 0.05$ and 1.0, with most estimates lying at the lower end of this range (Oey \& Clarke 1997; Oey, Clarke, \& Massey 2001; McClure-Griffiths et al. 2002; Oey \& García-Segura 2004; Ehlerová \& Palouš 2005). 
The term 'supershell' was first used by Heiles (1979) to describe atomic hydrogen shells with estimated formation energies of $E \gtrsim 10^{52} \mathrm{erg}$, but is now generally used loosely to refer to any large $(R \gtrsim 100 \mathrm{pc})$ shell-like structure. The theory of the formation of these superstructures via correlated supernovae and stellar winds was developed during the following years (e.g. McCray \& Snow 1979; Bruhweiler et al. 1980; Tomisaka, Habe, \& Ikeuchi 1981; Mac Low \& McCray 1988), and although alternative formation mechanisms have been proposed (e.g. Tenorio-Tagle 1981; Loeb \& Perna 1998; Wada et al. 2000), stellar feedback is successful in accounting for the observational characteristics, population-density, and energetics of the majority of objects (e.g. McCray \& Kafatos 1987; Ehlerova \& Palous 1996; McClure-Griffiths et al. 2002; Weisz et al. 2009; Warren et al. 2011). To the present day, a steady stream of observational work continues to catalogue and study new supershells both in the Milky Way and in external galaxies (see e.g. Kim et al. 1999; McClureGriffiths et al. 2002; Ehlerová \& Palouš 2005; Bagetakos et al. 2011).

\subsection{Evolution of a classical supershell}

The classical analytical model for a stellar wind bubble expanding into a uniform medium was derived by Weaver et al. (1977), and modified for a system formed by multiple stellar winds and supernovae by McCray \& Kafatos (1987). The early evolutionary stages of the system are dominated by stellar winds, which create a hot, low-density cavity into which subsequent supernovae inject their energy. By $t \sim 5 \times 10^{6} \mathrm{yr}$, these winds have switched off, and supernovae continue to inject energy until $t \sim 5 \times 10^{7} \mathrm{yr}$, with the mass in the cavity acting as a buffer to the supernova blast waves. During the stellar wind phase, the idealised bubble consists of an inner zone of hypersonic stellar wind, a shocked stellar wind layer, a shell of shocked ISM, and the ambient ISM. The system initially evolves adiabatically, expanding much faster than the radiative cooling timescales in any of the four zones, ending after several $10^{3}$ years, when radiative losses in the swept-up shell become important and it rapidly begins to cool. This drop in temperature is naturally accompanied by a drop in pressure, and the shell collapses to a thin, cool layer, compressed by the thermal pressure of the hot $\left(T \sim 10^{6} \mathrm{~K}\right)$ interior. The expansion continues to be driven by this thermal pressure, with a growth rate of the radius of the shell, $R$, given by $R \propto t^{3 / 5}$. This relation holds until such time as radiative cooling of the interior gas begins to become important, estimated to occur at several $10^{6}$ years for a typical clusterdriven superbubble (McCray \& Kafatos 1987). A shell whose interior thermal energy has been entirely radiated away will expand by conservation of momentum alone, with $R \propto t^{1 / 4}$. The shell may contain an ionised inner skin for much of its life, with a thickness determined by the radiation output of the remaining central sources.

\subsection{Disc blowout}

Shells with sufficient energy may expand rapidly along the disc vertical density gradient, eventually breaking out entirely and venting their hot interior gas into the halo. Accelerated vertical expansion leads to the growth of RayleighTaylor instabilities, culminating in the break-up of the shell and the release of its interior gas (Mac Low, McCray, \& Norman 1989; Tenorio-Tagle, Rozyczka, \& Bodenheimer 1990). The energy requirements for blowout depend sensitively on the structure of the local ISM into which the central cluster inputs its energy, as a well as on the distance of that cluster from the Galactic midplane, and the strength of the disc magnetic field (e.g. Tomisaka 1992, 1998). In a realistic, pre-structured medium, however, blowout almost certainly occurs relatively easily, as an expanding supershell seeks out low-density pathways through the inhomogeneous ISM (e.g. de Avillez \& Berry 2001; de Avillez \& Breitschwerdt 2005; Hill et al. 2012). These blown-out 'chimney' systems form a vital link from the disc to the halo, supplying the latter with the energy and metal-enriched material (Norman \& Ikeuchi 1989; see also review by Dickey 2012).

\subsection{The molecular ISM}

The central question in this review is the role played by largescale stellar feedback on the formation, destruction, and distribution of the molecular ISM. Figure 1 shows a cartoon of a supershell expanding in a stratified medium containing pre-existing molecular clouds, which illustrates both the large-scale features of the system's evolution and the range of effects it may have on the molecular ISM. An initially atomic shell may persist for long enough and accumulate sufficient material to become dense, cold, and molecular, eventually fragmenting to form discrete molecular clouds and new stars. A shell may also encounter existing dense clouds in its path, resulting in their dynamical disruption and eventual destruction. Such encounters are also quite capable of triggering star formation in pre-existing dense clouds, however, so that the formation and subsequent collapse of fresh molecular material is not the only route to star formation afforded by large-scale stellar feedback. While we do not focus on the question of triggered star formation in this review, it is prudent to point out that the 'destruction' of a molecular cloud cannot automatically be assumed to have a negative impact on star formation.

\section{MOLECULAR CLOUD FORMATION IN SUPERSHELLS: THEORY AND MODELLING}

An extensive body of literature exists on the physics and chemistry of the neutral ISM, and on the transition between its atomic and molecular phases. Here, we focus on that work which is of particular relevance to the formation of molecular clouds by large-scale stellar feedback-either through direct modelling of supershell systems or through the development 


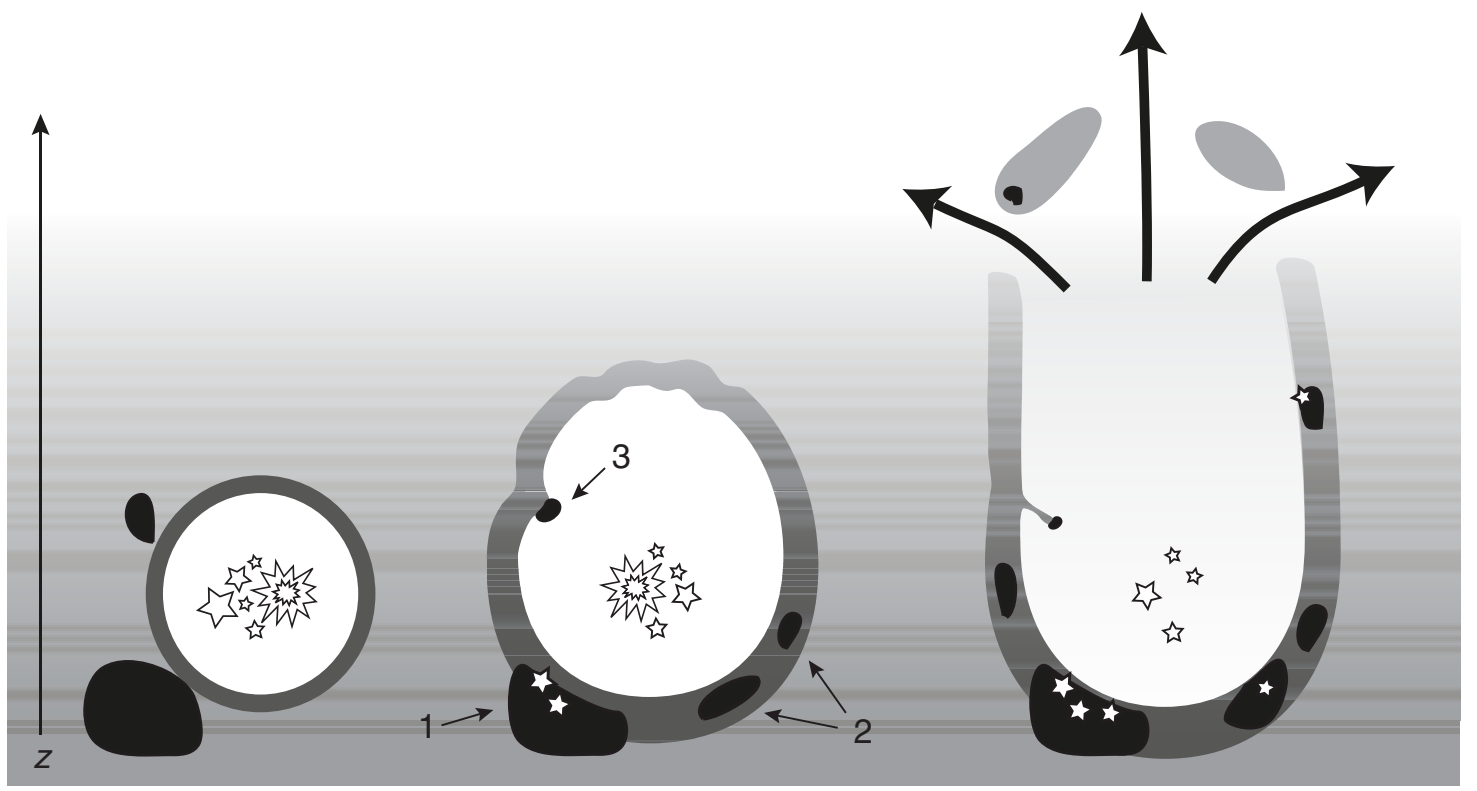

Figure 1. Cartoon showing an edge-on view of the evolution of a supershell in the Galactic plane (time sequence from left to right), illustrating some of the ways in which large-scale stellar feedback can affect the molecular ISM. Here, black clouds represent molecular gas and the greyscale is the ambient atomic ISM. Labels show examples of (1) the triggering star formation in existing molecular gas, (2) the formation of new molecular clouds, (3) the disruption and entraining of existing molecular clouds.

of general theory of ISM flows that includes those driven by supernovae and stellar winds.

\subsection{Molecule formation and destruction}

While recent work argues that the presence of molecules is not necessary for the formation of cold, dense gas (Glover \& Clark 2012; Mac Low \& Glover 2012), molecular emission lines are the primary tracer of the star-forming ISM, and an understanding of how the major species form and evolve is essential in interpreting observational data. From a theoretical perspective, a region of the ISM in which hydrogen is predominantly in the form of $\mathrm{H}_{2}$ constitutes a molecular cloud. However, observationally it is the abundance and properties of trace molecules-in particular $\mathrm{CO}$ - that determine whether a cloud is detectable.

Most treatments that explicitly follow molecule formation make use of approximate expressions for the formation rate of $\mathrm{H}_{2}$ on dust grains (e.g. Hollenbach \& Salpeter 1971; Tielens \& Hollenbach 1985). For conditions typical of the cold neutral medium $(\mathrm{CNM} ; T \sim 80 \mathrm{~K}, n$ $\sim 50 \mathrm{~cm}^{-3}$; see e.g. Field, Goldsmith, \& Habing 1969; Ferrière 2001), the characteristic formation rate is $\sim 3 \times 10^{7}$ $\mathrm{yr}$, but a strong density dependence ensures that $\mathrm{H}_{2}$ forms rapidly for densities that are even moderately enhanced with respect to canonical CNM values (see also Glover \& Mac Low 2011). For $\mathrm{H}_{2}$ to survive, it must be shielded from dissociating photons in the energy range $11.08<$ $h v<13.6 \mathrm{eV}$. While full modelling of $\mathrm{H}_{2}$ dissociation and shielding is complex (see e.g. van Dishoeck \& Black 1988; Draine \& Bertoldi 1996), a combination of efficient self- shielding and dust shielding means that for UV field strengths within a factor of few of the Draine field, hydrogen will typically be in its molecular form for visual extinctions of $A_{V} \gtrsim 0.1-0.5$ (e.g. Franco \& Cox 1986; van Dishoeck \& Black 1988; Bergin et al. 2004; Wolfire, Hollenbach, \& McKee 2010). This corresponds to column densities of roughly $\sim 1 \times 10^{21} \mathrm{~cm}^{-2}$ (Draine \& Bertoldi 1996).

For CO, the most commonly used line tracer of the molecular ISM, formation proceeds efficiently through collisional processes (van Dishoeck \& Black 1988), but less efficient self-shielding means that abundances are primarily determined by visual extinction (Glover \& Mac Low 2011). The shielding requirements are therefore somewhat more stringent than for $\mathrm{H}_{2}$, at $A_{V} \gtrsim 0.6-1.0$ (van Dishoeck \& Black 1988; Bergin et al. 2004; Wolfire et al. 2010). An interesting implication of this is the existence of a substantial quantity of 'dark' molecular gas not seen in the usual surveys (e.g. Grenier, Casandjian, \& Terrier 2005; Wolfire et al. 2010).

\subsection{Gravitational instability of expanding shells}

For molecular clouds to fulfil their role as star formation sites, they must become self-gravitating - or at least contain self-gravitating substructure. Motivated by this, a number of authors have applied the theory of gravitational instabilities in expanding shells to the parameter space appropriate to supershells. McCray \& Kafatos (1987) use an analytical approximation for the fragmentation of an expanding shell into gravitationally bound clouds to derive a time for the onset of gravitational instabilities as $t \approx 3.2 \times 10^{7} N_{*}^{-1 / 8} n_{0}^{-1 / 2}$ $a_{s}^{5 / 8} \mathrm{yr}$, where $N_{*}$ is the number of supernova progenitors in the parent cluster, $n_{0}$ is the density of the ambient medium 
and $a_{S}$ is the magnetosonic speed in the shell in $\mathrm{km} \mathrm{s}^{-1}$. For typical supershell parameters, this suggests a timescale of a few $10^{7}$ years for shells to fragment into gravitationally bound clouds. The authors also state that they expect supershells to become predominantly molecular well before this, within timescales of $\sim 10^{6} \mathrm{yr}$. However, this appears to be far too short for shells to accumulate sufficient material for $\mathrm{H}_{2}$ shielding, and is in general not consistent with other models or with observations.

A series of numerical models by Ehlerova et al. (1997), Efremov, Ehlerová, \& Palouš (1999), Ehlerová \& Palouš (2002), and Elmegreen, Palouš, \& Ehlerová (2002) use the thin shell approximation (Sedov 1959; Kompaneets 1960) to study the gravitational fragmentation of supershells over a broad range of parameter space, with particular focus on galactic environment. It should be noted that recent work by Dale et al. (2009) and Wünsch et al. (2010) has demonstrated that the thin shell approximation deviates from the predictions of numerical simulations in cases where the external confining pressure is small. Nevertheless, it provides a useful and computationally cheap method of exploring the large regions of parameter space appropriate for supershells evolving under a wide range of different conditions.

Ehlerova et al. (1997) note that fragmentation timescales are insensitive to the initial energy input, but are strongly density dependent, and assume a realistic input cluster size of 40-100 supernova progenitors to derive the condition that systems with $n_{0} \leqslant 0.3 \mathrm{~cm}^{-3}$ never become unstable. Similarly, Ehlerová \& Palouš (2002) derive a critical column density for gravitational fragmentation of $N \gtrsim 10^{20}-10^{21} \mathrm{~cm}^{-2}$ for realistic values of the energy input and sound speed in the ambient medium. Characteristic fragmentation timescales for solar neighbourhood densities of $n_{0} \sim 1 \mathrm{~cm}^{-3}$ are long, at $\sim 5 \times 10^{7}$ yr (Ehlerova et al. 1997), but would be significantly faster for moderately overdense regions. Similarly, in a stratified medium, it is the dense central regions within the Galactic plane that become unstable to gravitational collapse, while polar regions often remain stable throughout a shell's lifetime (see also Mashchenko \& Silich 1994). Here, the thickness of the disc is critical in determining the fate of the system, with Gaussian scale heights of $\sigma \lesssim 100 \mathrm{pc}$ resulting in shells that never fragment. Galactic rotation also strongly affects the evolution of the shell. Shear deforms shells into elongated ellipses, and mass accumulated in the shell slides to the tips, forming instability regions there. This phenomenon has also been noted by Tenorio-Tagle \& Palous (1987), who demonstrate that in a typical Milky Way environment these sites can accumulate sufficient matter to go molecular and form giant molecular clouds (GMCs). The scenarios explored in these studies imply that the type of galaxy into which a supershell is born strongly influence its propensity for gravitational instability, and hence the likelihood of forming new molecular clouds through this mechanism. Elmegreen et al. (2002) derive a set of dimensionless conditions for this 'triggering' and find that dwarf galaxies such as the LMC have an advantage over early-type spirals such as the Milky Way.

\subsection{Molecular cloud formation in colliding flows}

A realistic, turbulent ISM is subject to a range of processes that are not treated in the simplified gravitational stability analysis above, yet which may have a profound effect on its evolution. The last 10-15 years have seen a profusion of hydrodynamic and magnetohydrodynamic (MHD) simulations that attempt to model the ISM in increasingly realistic ways, many focussing on the formation of molecular clouds.

Much work centres around the paradigm of flow-driven molecular cloud formation, in which the production of starforming molecular gas is integrated into the modern picture of a dynamic, turbulent ISM. In this picture, the compression, cooling, and fragmentation of the atomic medium in colliding ISM flows produces clumpy sheets and filaments of cold, turbulent material, which go on to become highly structured, self-gravitating molecular clouds once density and column density requirements are met (see review by Vázquez-Semadeni 2010). Hartmann et al. (2001) lay out part of the astrophysical motivation for this picture, which has its roots in observational evidence that molecular clouds must form rapidly, birth stars rapidly, and disperse rapidly (see also Elmegreen 2000; Hartmann 2003). These authors use simple analytical approximations to argue that material accumulated in large-scale ISM flows will become molecular, magnetically supercritical and self-gravitating on roughly similar timescales, and that supershells formed by stellar feedback are excellent candidates for the drivers of such flows.

A combination of thermal and hydrodynamical instabilities, together with turbulent compression (see e.g. Federrath et al. 2010), is critical in condensing cold gas from the warm ambient medium. The atomic ISM is a thermally bistable medium (Field et al. 1969; Wolfire et al. 1995), with two linearly stable phases corresponding to the warm neutral medium (WNM) and CNM - the latter being the atomic precursor to molecular clouds. Trans-sonic converging flows of initially stable WNM trigger runaway cooling and the formation of cold gas (Hennebelle \& Pérault 1999), with dense structures developing rapidly on sub-parsec scales (e.g. Heitsch et al. 2006; Vázquez-Semadeni et al. 2006). Models have progressed to include increasingly realistic physics and explore various different aspects of the cloud formation process, including the roles of turbulence (Audit \& Hennebelle 2005; Vázquez-Semadeni et al. 2006; Glover \& Mac Low 2007), magnetic fields (Hennebelle et al. 2008; Inoue \& Inutsuka 2008; Banerjee et al. 2009; Heitsch, Stone, \& Hartmann 2009; Inoue \& Inutsuka 2009), molecular chemistry (Bergin et al. 2004; Glover \& Mac Low 2011; Clark et al. 2012; Glover \& Clark 2012; Inoue \& Inutsuka 2012), self-gravity (Vázquez-Semadeni et al. 2007; Heitsch, Hartmann, \& Burkert 2008a; Heitsch et al. 2008b; Hennebelle et al. 2008), and the interplay between different instabilities (Heitsch et al. 2008a).

Taken together, these simulations suggest that for expansion velocities and ambient densities typical of Galactic 
supershells $\left(v_{\exp } \sim 10-20 \mathrm{~km} \mathrm{~s}^{-1}\right.$ and $\left.n_{0} \sim 1-5 \mathrm{~cm}^{-3}\right)$, substantial quantities of CO-rich molecular gas can be produced on timescales of a few $10^{6}$ to $\sim 10^{7} \mathrm{yr}$ (Bergin et al. 2004; Heitsch \& Hartmann 2008; Clark et al. 2012; Inoue \& Inutsuka 2012), provided that magnetic fields do not provide significant support against collapse (Inoue \& Inutsuka 2008, 2009; Heitsch et al. 2009). For the parameter space appropriate to the atomic ISM, cooling timescales are much shorter than the characteristic timescales for gravitational instability, and thermal/dynamical instabilities dominate over gravity in driving fragmentation in the compressed medium (Heitsch et al. 2008a). This implies that small, dense, thermally driven condensations will develop long before a supershell becomes gravitationally unstable, and that some molecular gas can form without the ISM becoming self-gravitating (Heitsch \& Hartmann 2008). However, global contraction helps to reduce overall timescales, and in particular may be important in accumulating sufficient material to shield $\mathrm{CO}$ so that clouds become observable (Heitsch \& Hartmann 2008). Once initiated, gravitational collapse will proceed hierarchically, beginning first in the dense substructures already imprinted on the medium by thermal instabilities and turbulence (Heitsch et al. 2006; Vázquez-Semadeni et al. 2007). One implication of this is that clouds are expected to begin forming stars rapidly once they become visible in molecular tracers, suggesting that the triggered formation of molecular clouds is synonymous with the triggered formation of stars.

These results provide a strong theoretical basis for molecular cloud formation in supershells and demonstrate its viability in systems with flow properties that are consistent with observed objects. The next stage is to explicitly simulate supershell systems as opposed to generalised flows. So far only one high-resolution hydrodynamical simulation has explicitly modelled flow-driven molecular cloud formation in a supershell system. Ntormousi et al. (2011) present two-dimensional (2D) models of two superbubbles expanding into a uniform homogeneous or turbulent medium, and investigate the formation of dense gas around the bubble peripheries and interaction zone. Their supershells are blown by supernovae and time-dependent stellar winds, with properties calculated from population synthesis models, and evolve with realistic flow velocities, timescales, and size scales. Figure 2 reproduces their snapshot of the temperature and density of the turbulent model at times of 3 and $7 \mathrm{Myr}$. By the end of a 7-Myr run, their simulation box is filled almost entirely by the shell systems, and a combination of nonlinear thin shell, thermal, and Kelvin-Helmholz instabilities has lead to the formation of copious amounts of clumpy and filamentary cold $(T<100 \mathrm{~K})$ gas - mostly associated with the shell collision zone. They find that $\sim 65 \%-85 \%$ of the gas is contained in these small, dynamical structures, which have characteristic sizes of $\lesssim 1 \mathrm{pc}$ and densities of $10^{2}-10^{3} \mathrm{~cm}^{-3}$-fulfilling the approximate density and column density requirements for molecule formation.

The 2D nature of these simulations imposes some limitations on the evolution and structure of the turbulence and fragmentation. Unlike in 3D models, where large-scale structures break up into smaller fragments, in 2D simulations they have a tendency to merge into larger agglomerations (e.g. Federrath et al. 2010), potentially affecting the ease with which dense clouds are formed. Conversely, the lack of selfgravity in the simulations is expected to have the opposite effect-making it harder to form dense clouds. The inclusion of magnetic fields in future models would also modify the properties of the collision zone (e.g. de Avillez \& Breitschwerdt 2005) and likely provide additional support. Nevertheless, these models are a promising first step, and will be developed extensively over the coming years to include more realistic physics.

Finally, it is likely that both pre-existing inhomogeneities in the ISM and magnetic field orientation lead to a selection effect for molecular cloud formation in supershell walls. A moderate elevation in the mean density along a swept-up column significantly reduces cooling and molecule formation timescales, suggesting that cloud formation sites may be determined by the placement of pre-existing concentrations of moderately overdense gas such as CNM sheets or filaments (see also Dobbs, Pringle, \& Burkert 2012 for similar findings on galactic scales). Conversely, magnetic pressure has the power to completely prevent the formation of molecular gas when a significant component of the field exists perpendicular to the flow direction, leading to the requirement that this perpendicular component be vanishingly small (Inoue \& Inutsuka 2009; Heitsch et al. 2009). While it remains to be seen how the inclusion of self-gravity and non-ideal MHD processes affects the rigidity of this conclusion, the selection of cloud formation sites by field orientation and mean density may provide an attractive explanation for why real supershells are not more molecular than they are.

\subsection{Whole-disc models of the feedback-structured ISM}

While high-resolution numerical simulations have examined in detail the small-scale processes associated with gas cooling and fragmentation, another class of model tackles the global effects of stellar feedback on the structure and dynamics of the ISM. Such simulations generally model kpc-scale sections of galaxy disc at moderate resolution with realistic supernova input rates, and have been very successful in producing the cold, warm neutral, warm ionised, and hot components of the ISM with reasonable mass fractions, distributions, and structure. Salient features include a thin cold disc, a 'frothy' disc of warmer material, and the existence of a great many shells, supershells and their broken fragments with 'tunnels' channeling their hot interior gas out into the halo (e.g. de Avillez \& Berry 2001; de Avillez \& Breitschwerdt 2005; Joung \& Mac Low 2006; Joung et al. 2009; Hill et al. 2012). An example is shown in Figure 3, from Hill et al. (2012). While these models lack the resolution to properly resolve very dense gas formation, the fact that the bulk of the cold material is concentrated into high density clouds in 

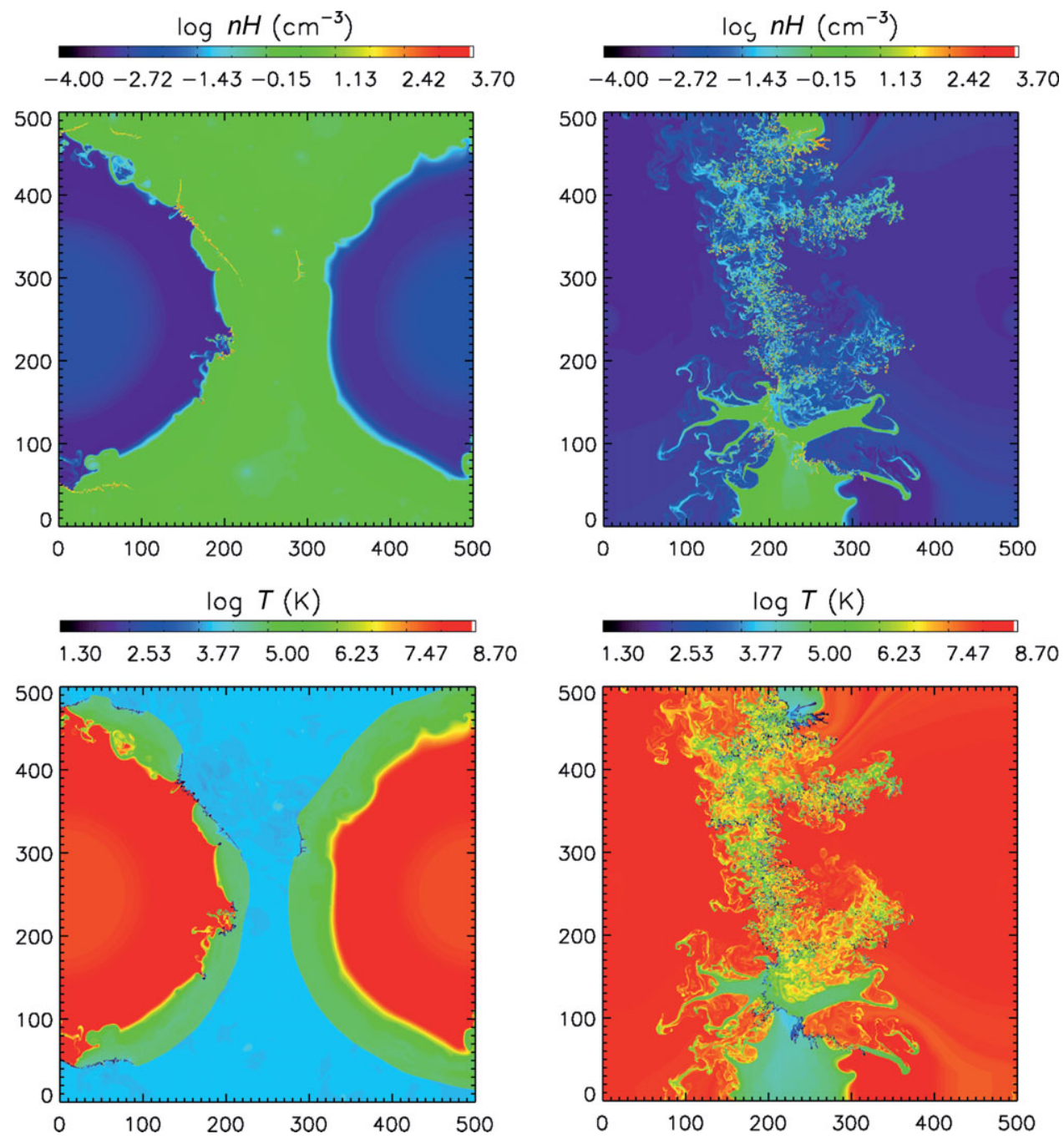

Figure 2. High-resolution, 2D hydrodynamical simulations of cold gas formation at the interface of two superbubbles colliding in a turbulent diffuse medium (reproduced by permission of the AAS from Ntormousi et al. 2011). These snapshots show the evolution of the system at $3 \mathrm{Myr}$ (left) and $7 \mathrm{Myr}$ (right) after the start of the simulations. The top panels show hydrogen number density and the bottom panels show gas temperature. After 7 Myr, copious amounts of clumpy and filamentary cold gas have been formed.

shock-compressed layers around or between bubbles (e.g. de Avillez \& Breitschwerdt 2005) is highly consistent with the scenario described in Section 3.3.

The lack of self-gravity and realistic disc dynamics mean that while the above models are a promising demonstration of the feasibility of molecular cloud formation in stellar feedback flows, they are unable to address the critical question of its relative importance in a galactic context. Simulations of rotating, self-gravitating galaxy discs provide a more complex picture, in which large-scale gravitational instabilities and spiral arms play a major role in dense gas formation, and the contribution of stellar feedback is by no means clear (e.g. Wada \& Norman 2001; Shetty \& Ostriker 2008; Bournaud et al. 2010; Tasker 2011; Dobbs et al. 2011, 2012). Recently, Dobbs et al. (2012) have explicitly addressed the question of which mechanisms are responsible for driving the compressive motions that form the GMCs in their simulations, and find that the primary drivers depend on the properties of their model galaxies, such as the nature of the spiral potential and the level of star formation feedback. While conclusions remain open, a future in which these predictions can be directly compared with observations is promising, as new facilities such as the Atacama Large Millimeter/submillimeter Array (ALMA) come online.

\section{PRE-EXISTING MOLECULAR CLOUDS}

Supernovae and stellar winds propagate into a highly structured, inhomogeneous medium that already contains dense gas. The effects of cluster feedback on existing molecular clouds range from the violent ionisation, compression, and dissipation of the parent cloud at early evolutionary stages, to the weak interaction of a cloud with an old, almost-stalled supershell. The question of how feedback affects parent 


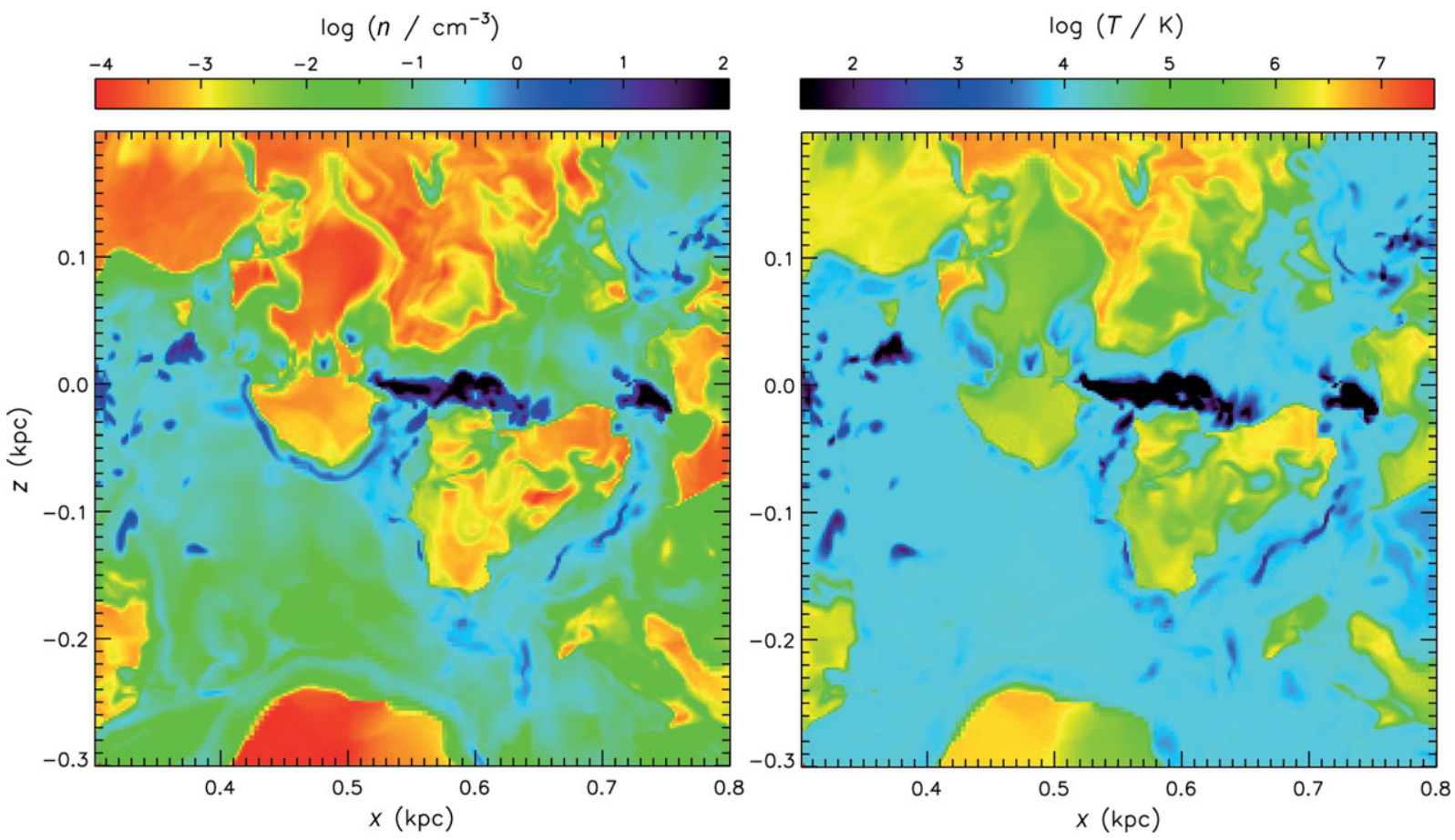

Figure 3. Images of density and temperature from MHD simulations in which supernovae drive turbulence and establish a multi-phase, stratified medium (reproduced with permission from the erratum to Hill et al. 2012). The images are 2D slices through a 3D simulation, where the $x$ axis is in the midplane and the $z$ axis shows the vertical distance from the midplane. The model used here is the magnetised model with 2-pc resolution in the midplane ('bx50hr') described by Hill et al. (2012). Energy injected by supernovae creates hot, low density remnants, surrounded by dense, long-lived filaments of cold gas.

molecular clouds is a complex one, involving the combined effects of strong ionisation, radiation pressure, and mechanical energy input on a pre-structured cloud. The interplay between these processes and the importance (or not) of triggered star formation is still a topic of much debate and will not be dealt with in this review (see e.g. Dale, Ercolano, \& Bonnell 2012; Walch et al. 2012, and references within). Instead, we focus primarily on the region of parameter space appropriate to a system that has expanded far beyond its birth site and out into the surrounding medium-i.e. an object that would already be defined as a 'supershell' by an observer. For an idealised shell of $R \gtrsim 50 \mathrm{pc}$, this implies expansion velocities of no greater than a few tens of $\mathrm{km} \mathrm{s}^{-1}$ (McCray \& Kafatos 1987), translating to shocks that are mildly supersonic in the WNM.

\subsection{Cloud disruption}

The dynamical interaction of a dense cloud with a shocked flow is commonly parameterised in terms of the "cloud crushing time', $t_{\mathrm{cc}}=\left(r_{0} / v_{i}\right)\left(n_{\mathrm{cl}} / n_{i}\right)^{0.5}$, where $r_{0}$ is the cloud radius, $v_{i}$ is the velocity of the shock in the ambient medium, and $n_{\mathrm{cl}}$ and $n_{i}$ are the number densities of the cloud and the ambient medium, respectively. The simplest case is a steady planar strong shock and a smooth spherical cloud, in which radiative losses, thermal conduction, gravity and magnetic fields are neglected. Such clouds are significantly disrupted within a few $t_{\mathrm{cc}}$, on a destruction timescale $t_{\text {dest }}$, defined as the time taken for the core mass of the cloud to reduce be a factor of 1/e (Klein, McKee, \& Colella 1994; Nakamura et al. 2006). For the test case of a small $\left(r_{0}=2 \mathrm{pc}\right)$, moderately dense $\left(n_{\mathrm{cl}}=200 \mathrm{~cm}^{-3}\right)$ cloud encountering a gently expanding $\left(v_{i}=20 \mathrm{~km} \mathrm{~s}^{-1}\right)$, thick, dense $\left(n_{i}=10\right)$ shell, $t_{\mathrm{cc}} \approx 0.4 \mathrm{Myr}$, implying $t_{\text {dest }} \leqslant 2-3 \mathrm{Myr}$.

In reality, this is likely a lower limit to cloud survival times. The Mach numbers of supershell shocks are relatively low $\left(\mathcal{M} \sim 2.5\right.$ for a $20 \mathrm{~km} \mathrm{~s}^{-1}$ shock in the WNM), and timescales may be several times longer than the strong shock case (Nakamura et al. 2006; Pittard, Hartquist, \& Falle 2010). Radiative cooling also cannot be ignored for supershell-molecular cloud interactions. Its inclusion inhibits cloud destruction, encourages the formation of overdense clumps and filaments, and can significantly extend timescales above the adiabatic limit (Mellema, Kurk, \& Röttgering 2002; Fragile et al. 2004; Orlando et al. 2005). The role of magnetic fields is more complex - while they tend to inhibit the development of hydrodynamic instabilities and reduce mixing, both orientation and field strength are important, and some configurations may result in more efficient fragmentation of the cloud material (Gregori et al. 1999; Orlando et al. 2008; Shin, Stone, $\&$ Snyder 2008). Conversely, environmental turbulence has been shown to speed up cloud destruction, though the magnitude of the effect is sensitive to the properties of the assumed turbulence and is still under investigation (Pittard et al. 
2009, 2010). Nevertheless, it seems reasonable to assume that dynamical disruption of molecular clouds by an evolved shell proceeds reasonably slowly, on timescales comparable to the shell lifetime. It is worth noting, however, that the physical stripping and dissipation of cloud material also renders a disrupted cloud increasingly vulnerable to the UV dissociation of $\mathrm{CO}$. The observable lifetime of the entity we see as a $\mathrm{CO}$ cloud may therefore be shorter than the survival time of the dense material itself (see also Dawson et al. 2011b).

The efficiency of momentum transfer is also important, because it determines how readily the shocked cloud can be accelerated by the expanding shell. Adiabatic models find that characteristic drag timescales are generally comparable or lower than $t_{\text {dest }}$, suggesting that a shocked cloud-or its remaining fragments-will undergo significant acceleration before being completely disrupted (Klein et al. 1994; Nakamura et al. 2006). Higher density contrasts and lower Mach numbers result in less efficient acceleration (e.g. Pittard et al. 2010), as does any process that reduces the surface area of the cloud perpendicular to the shock direction (e.g. radiative cooling; Orlando et al. 2008), while environmental turbulence and certain magnetic field orientations may increase it (Shin et al. 2008; Pittard et al. 2009). As in the case of cloud destruction, the details of the cloud acceleration depend both on the model physics and on the cloud-shock parameters, and the final velocity of the accelerated cloud material may be anywhere between $\sim 0.1$ and 0.8 times the initial shock velocity.

The majority of these studies assume that the post-shock flow has an infinite depth - a situation that is clearly inappropriate for the shell-cloud case. The finite depth of a swept-up shell limits the time of the flow-cloud interaction, and a cloud encountering a supershell will likely pass into the hot shell interior before it is destroyed, leaving a tail of shell material trailing behind it (Pittard 2011). For a shell thickness of $\sim 10 \mathrm{pc}$, the example cloud described above will enter the interior on timescales of $\sim 1$ to several Myr, depending on the drag efficiency. In the interior regime, the material flowing past it will be more diffuse and less dynamically disruptive, but will likely be hot ionised medium. The classical evaporation timescale for clouds embedded in fully ionised medium is given by $t_{\text {evap }} \sim 3.3 \times 10^{20} n_{\mathrm{cl}} T^{-5 / 2}{ }_{i} r_{\text {cl,pc }}^{2}$ yr (Cowie \& McKee 1977), which equates to $\sim 10^{8}$ yr for the example cloud, assuming $T_{i}=10^{6} \mathrm{~K}$. This suggests that thermal evaporation is unlikely to be a dominant destructive influence.

Finally, while we do not deal explicitly in this review with the topic of triggered star formation, it should be stressed that the destruction of a molecular cloud cannot be assumed to have a negative impact on star formation. Indeed, for moderate shock velocities appropriate to supershell systems, star formation may be triggered readily for a range of molecular cloud properties (e.g. Vanhala \& Cameron 1998; Melioli et al. 2006; Leão et al. 2009). These considerations are important in interpreting observations of supershell-associated molecular gas and stars.

\section{OBSERVATIONS OF MOLECULAR GAS IN SUPERSHELLS}

\subsection{General considerations}

In this section, we will review observational evidence for molecular cloud formation in supershells, as well as some broader examples of the interaction between stellar feedback and the molecular ISM.

The most commonly used spectral line tracers of the molecular ISM are the low- $J$ transitions of CO. CO is the workhorse of molecular ISM astronomy, since its high abundance $\left(n_{\mathrm{CO}} / n_{\mathrm{H}_{2}} \sim 10^{-4}\right)$, low-lying rotational energy levels $(\Delta E / k \sim 5 \mathrm{~K}$ for $J=1)$, and relatively low critical densities ( $n \sim 1000 \mathrm{~cm}^{-3}$ for the lower $J$ transitions) mean that it is readily observable even in relatively diffuse, quiescent molecular gas. For a detailed physical and chemical census of a molecular cloud, it is possible to assemble a suite of lines from multiple tracers that probe a wide range of density and temperature regimes, an approach that is particularly useful for detailed studies of the star formation process. However, from a molecular cloud formation perspective, we are generally more concerned with identifying zones in which the ISM is simply in its molecular form. $\mathrm{CO}$ is an excellent choice for this, although it is worth noting that even $\mathrm{CO}$ fails to trace the most diffuse molecular gas, where hydrogen is in the form of $\mathrm{H}_{2}$ but carbon is atomic (e.g. Reach, Koo, \& Heiles 1994; Grenier et al. 2005; Wolfire et al. 2010).

Molecular gas occupies only a small volume fraction of the ISM, and probes of other physical regimes are needed to form a complete picture of a supershell system (see e.g. Heiles, Haffner, \& Reynolds 1999). H I observations in particular are indispensable in probing the structure and kinematics of the neutral ISM, and in correctly relating molecular clouds to the global structure of a supershell system. Much of the work discussed below combines $\mathrm{H}$ I and $\mathrm{CO}$ data to investigate the structure and evolution of the ISM in supershells. Other tracers such as $\mathrm{H} \alpha$ and soft $\mathrm{X}$-rays are also useful, and provide valuable information on the ionised inner walls and the hot diffuse medium in shell interiors.

A key morphological/kinematic indicator of potential cloud formation is co-moving $\mathrm{CO}$ clouds that form coherent parts of an atomic shell-either lying along $\mathrm{H}$ I walls or well embedded within them. The converse is molecular clouds that show a head-tail morphology or other signs of dynamical disruption, or are entrained in a shell interior, likely indicating pre-existing dense gas. This simple diagnostic is by no means perfect. Various hybrid scenarios in which molecular clouds are formed early in a system's evolution and later impacted by subsequent supernova blast waves are certainly possible. Moreover, analysis can be frustrated by the irregularity of real observed supershells and line-of-sight confusion. Nevertheless, morphological diagnostics are an excellent starting point.

A complementary approach is to measure whether supershells are associated with a net increase in the molecular 
fraction of the ISM in the volumes they occupy. This attempts to answer the question of whether the formation of new molecular gas has dominated over the destruction of pre-existing clouds, as measured at a particular epoch in the system's evolution. This is an approach taken by Dawson et al. (2011b, 2013, discussed below) by comparing the $\mathrm{H}_{2}$ mass fraction in supershell volumes with nearby control regions.

Finally, stellar population studies are a powerful tool for reconstructing the history of a system. As well as providing valuable constraints on the original energy input from the central cluster, they can also provide insight into the nature and timing of any triggering that may have occurred during a supershell's evolution.

\subsection{The local ISM}

The local ISM, within a few hundred parsecs of the Solar system, is in many ways a microcosm of much of the Milky Way disc. It is an environment that has been carved out and shaped by multiple generations of stellar activity - characterised by a complex structure of loops, filaments, tunnels, irregular shells, and bubbles; and populated by the stellar clusters and associations variously responsible for and triggered by this activity (see de Geus 1992; Heiles 1998; Lallement et al. 2003; Frisch, Redfield, \& Slavin 2011). Unsurprisingly, the distribution of the local molecular ISM is closely related to these superstructures.

Perhaps the most striking example is the Gould Belt and its $\mathrm{H}$ I counterpart the Lindblad Ring. The Gould Belt/Lindblad Ring is the expanding, inclined ring of neutral gas and starforming regions that contains all of the major local molecular cloud complexes within a distance of $\sim 500 \mathrm{pc}$ from the Sun, including Orion, Taurus, Perseus, Ophiuchus, and Lupus (Taylor, Dickman, \& Scoville 1987; see also review by Poppel 1997). If the Gould Belt is a feedback structure, it is a superb candidate for the formation of molecular gas and stars from a swept-up supershell. Indeed, one scenario holds that the Gould Belt clouds are the remnants of an old fossil supershell that has fragmented sometime in the last 15-25 Myr to form gravitationally bound star-forming complexes (Olano 1982; see also Bally 2001). However, while stellar feedback is a strong contender, the origin of the Gould Belt is unclear, with other candidates including the impact of a high-velocity cloud (Comeron \& Torra 1992, 1994) and the breaking of a supercloud entering the spiral arm (Olano 2001; see also Grenier 2004 and references therein).

As this uncertainty suggests, reconstructing a region's history is often a challenging exercise in astronomical forensics. Nevertheless, there are numerous indications that stellar feedback has played an important role in the evolution of the local molecular ISM. We will explore a few illustrative examples in the paragraphs to follow.

The Lupus and Ophiuchus molecular cloud complexes form part of the neutral ISM delimiting the cavity of the Local Bubble-the old supershell in which the Sun is cur- rently located (see Lallement et al. 2003). Their distances are $\sim 155$ and $\sim 120 \mathrm{pc}$, respectively (Lombardi, Lada, \& Alves 2008), and both complexes have estimated (CO-bright) molecular masses of $\sim 10^{4} \mathrm{M}_{\odot}$ (de Geus \& Burton 1991; Tachihara et al. 2001), meaning that they are smaller than typical GMCs. The Lupus clouds are located between the Upper-Centaurus-Lupus and Upper-Sco subgroups of the Sco-Cen OB association, apparently lying on the edge of an $\mathrm{H}$ I shell blown by the latter (and younger) of these (de Geus 1992). Parts of the cloud complex show evidence of interaction with the expanding shell, and it has been suggested that star formation may have been triggered by both the current interaction and a previous shock wave from the older Upper-Centaurus-Lupus shell several Myr ago (Tachihara et al. 2001; Merín et al. 2008; Tothill et al. 2009). An implication of this is that the molecular matter-or at least some of it - pre-dated these shells. Along similar lines, the detailed work on the Ophiuchus region by de Geus (1992) stresses the interaction of the Upper-Sco subgroup with the much-studied $\rho$-Oph cloud. Here, molecular gas clearly predates the current interaction, and is being stripped from the cloud to form streamers and tails. However, he also suggests the possibility of in situ formation in the shell walls for some other parts of the complex. This is consistent with an alternative scenario suggested by Preibisch \& Zinnecker (2007), in which feedback flows formed both the present Lupus and Ophiuchus complexes, and the Lupus clouds were formed sandwiched at the interface where the two shells meet. This scenario is attractive in that it does not require molecular gas to exist for an unrealistically long time prior to the onset of star formation. The authors also stress the role of multiple episodes of energy input, with clouds initially being formed during the wind bubble stage and then swept over to trigger star formation during a later supernova phase. It is interesting to note that this is also a solution proposed by Inoue \& Inutsuka (2009) to overcome the difficulty of achieving high enough densities for molecular gas and star formation in a magnetically supported cloud.

Another interesting Gould Belt complex is the Cepheus Flare. This region contains $\sim 5 \times 10^{3} \mathrm{M}_{\odot}$ of molecular gas that forms part of an expanding shell of $R \sim 50$ pc enclosing an old supernova remnant (SNR) (Grenier et al. 1989; Olano, Meschin, \& Niemela 2006). Kun (1998) notes that the location of star-forming sites at the edges of the $\mathrm{CO}$ clouds suggests external shock triggering, which Olano et al. (2006) attribute to the same energetic event that created the shell-again, tentatively suggesting that the clouds pre-date the current interaction. Interestingly, this shell may be associated with the prominent $\mathrm{H}$ I ring known as the North $\mathrm{Ce}$ lestial Pole Loop (Meyerdierks, Heithausen, \& Reif 1991), which itself contains the well-known molecular cirrus cloud, the Polaris Flare (Heithausen \& Thaddeus 1990). The Polaris Flare is a classic example of a translucent molecular cloud-diffuse (Meyerdierks \& Heithausen 1996), gravitationally unbound (Heithausen \& Thaddeus 1990), and nonstar-forming (André et al. 2010). It has been described as 
'the archetype of the initial phases of molecular cloud formation' (Miville-Deschênes et al. 2010). In this context, it is especially interesting to note that the Polaris Flare may be material swept up by the Cepheus Flare shell.

Somewhat further afield, Heiles (1998) suggests a scenario in which star formation in the Orion and Gum regions (both at $D \sim 500 \mathrm{pc}$ ) was triggered by a more distant supershell GSH $238+00+09$, although it is unclear whether molecular clouds were formed by the action of the shell or merely compressed by it. This secondary star formation later blew the Orion-Eridanus (e.g. Heiles et al. 1999) and Gum Nebula (e.g. Yamaguchi et al. 1999a) bubbles, both of which are themselves associated with CO-bright gas, including many cometary globules-presumably remnants of the parent clouds of the new young clusters (see Yamaguchi et al. 1999a, and references therein).

\subsection{The Milky Way}

In the wider Milky Way, a large number of studies have documented molecular gas associated with supershells (Kundt \& Mueller 1987; Koo \& Heiles 1988; Heiles, Reach, \& Koo 1996; Maciejewski et al. 1996; Normandeau, Taylor, \& Dewdney 1996; Jung et al. 1996; Patel et al. 1998; Rizzo \& Arnal 1998; Yamaguchi et al. 1999a; Fukui et al. 1999; Kim \& Koo 2000; Carpenter, Heyer, \& Snell 2000; McClureGriffiths et al. 2000; Matsunaga et al. 2001; Moriguchi et al. 2002; Megeath et al. 2003; Moór \& Kiss 2003; Dawson et al. 2008b, 2011b), including notable examples of multiple generations of sequential star formation triggered by large-scale stellar feedback (Patel et al. 1998; Oey et al. 2005). However, as in the case of the local ISM, there is often ambiguity surrounding the origins of the molecular material itself. Below we will discuss those objects for which the issue has been explicitly considered.

The Perseus Chimney is a vertically elongated $(\sim 110 \times$ $230 \mathrm{pc}$ ) supershell in the Perseus arm of the outer Galaxy, which is likely in the process of blowing out of the Galactic disc (Normandeau et al. 1996; Dennison, Topasna, \& Simonetti 1997; Basu, Johnstone, \& Martin 1999). The shell is bordered on its lower edge by the bright W3/W4 H II regions, and is associated with substantial quantities of molecular gas, particularly around its base in the Galactic plane (Digel et al. 1996; Heyer \& Terebey 1998). Heyer et al. (1996) and Taylor et al. (1999) report on two cometary molecular clouds embedded in the chimney cavity, at altitudes of 20 and $50 \mathrm{pc}$ and with molecular masses of $\sim 5 \times 10^{3}$ and $\sim 3 \times 10^{3} \mathrm{M}_{\odot}$. These clouds show strong evidence of interaction with the stellar winds and UV radiation of the IC 1805 cluster at the chimney base, including streamers and tails of both $\mathrm{H}$ I and $\mathrm{CO}$ pointing directing away from the ionising sources. They are both well interpreted as remnants of an original molecular complex that was destroyed in the formation of the chimney. For the large $\left(\sim 10^{5} \mathrm{M}_{\odot}\right.$; Digel et al. 1996) reservoir of molecular gas around the base of the supershell, interpretations are less clear. Star formation along the edge of the shell in material bordering the $\mathrm{W} 4$ region implies compression and triggering, with the most natural explanation being that this occurs in pre-existing molecular gas (Carpenter et al. 2000; Oey et al. 2005). However, whether all of this material represents remnants of an older GMC complex or whether additional episodes of compression have supplemented the molecular gas reservoir in the past is not clear. Some hint that this might have been the case comes from the suggestion of Oey et al. (2005) that the IC 1805 cluster itself may represent a later generation of star formation. In this picture, its formation was triggered by an older progenitor cluster responsible for blowing an even older and larger supershell of which the current Perseus Chimney forms only the lower part (Reynolds et al. 2001).

Several other studies have considered molecular cloud formation in a more quantitative way. Jung et al. (1996) report the association of a large mass of molecular gas $\left(\sim 1.1 \times 10^{6} \mathrm{M}_{\odot}\right)$ with the outer Galaxy supershell GS 234 2 , which they interpret in the context of the gravitational fragmentation of the swept-up atomic medium based on the analytical expression of McCray \& Kafatos (1987). Kim $\&$ Koo (2000) make a more careful analysis of two small $\left(\sim 10^{3} \mathrm{M}_{\odot}\right)$ molecular clouds well embedded within the $\mathrm{H} \mathrm{I}$ of the 'Galactic Worm' GW 46.4+5.5, which makes up the vertical wall of a large $(340 \times 540 \mathrm{pc})$ and relatively local $(D \sim 1.4 \mathrm{kpc}) \mathrm{H}$ i shell. They also use the analytic expression for gravitational fragmentation, and find that the timescale for its onset is approximately equal to the estimated kinematic age of the shell ( $t \sim 5 \mathrm{Myr}$ ), leading them to conclude that the molecular gas has likely formed from the sweptup ambient medium. In this context, it is interesting that the molecular clouds (as measured from CO) do not appear to be globally gravitationally bound. While the molecular portion of the ISM presumably does not account for the entire mass of the 'fragment' in which it is found, it is worth noting that unbound clouds are not inconsistent with the predictions of the colliding flows picture of molecular cloud formation.

\subsubsection{GSH 287+04-17 and GSH 277+00+36: Detailed case studies and quantitative analysis}

The most detailed analysis of the origin and evolution of molecular gas in Milky Way supershells is given in a series of papers by Dawson et al. (2008a, 2008b, 2011a, 2011b). These authors present matched resolution, parsec-scale observations of $\mathrm{H}$ I and $\mathrm{CO}$ in two Galactic supershells, GSH 287+04-17 and GSH 277+00+36, enabling detailed investigation of the relationship between the atomic and molecular ISM in shell walls. They find substantial quantities of co-moving molecular gas in the $\mathrm{H}$ I shells, with rich substructure in both tracers, including molecular gas seen elongated along the inner edges of the atomic walls, embedded within atomic filaments and clouds, or taking the form of small $\mathrm{CO}$ clouds at the tips of tapering atomic 'fingers'. Figure 4 shows a section of the wall of GSH $287+04-17$ that illustrates these features. Small CO clouds at the tips of $\mathrm{H}$ I fingers have no 


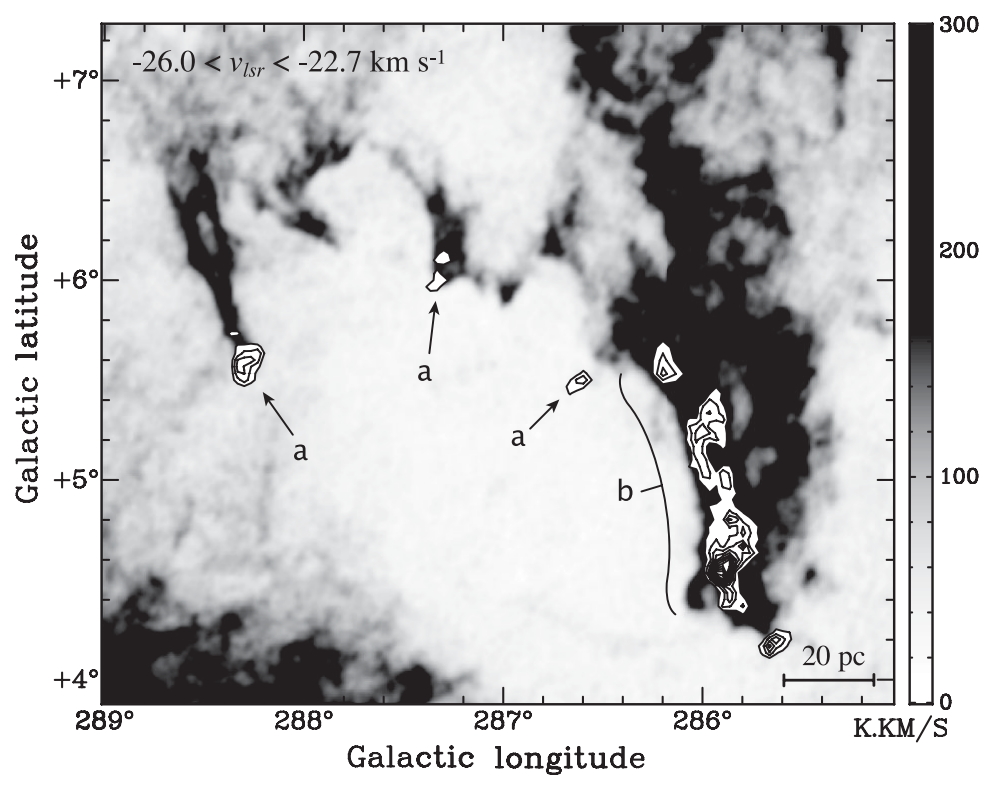

Figure 4. Subsection of the wall of the Galactic supershell GSH 287+04-17 integrated over the velocity range indicated in the top-left corner of the image (Reproduced from Dawson et al. 2012). The greyscale is $\mathrm{H}$ I and the filled contours are ${ }^{12} \mathrm{CO}(J=1-0)$. Features labelled ' $\mathrm{a}$ ' indicate small $\left(M_{\mathrm{H}_{2}} \sim 10^{2-3} \mathrm{M}_{\odot}\right)$ clumps of molecular gas offset towards the tips of atomic ÔfingersÕ that point in the direction of the shell centre. These are $\mathrm{CO}$ clouds that are likely being destroyed by their interaction with the shell. The feature labelled ' $\mathrm{b}$ ' is an example of a larger $\left(M_{\mathrm{H}_{2}} \sim 10^{4} \mathrm{M}_{\odot}\right)$ molecular cloud that is well embedded in atomic material and forms a coherent part of the main shell wall. This is a strong candidate for in situ formation of molecular gas in the shell wall. This entire region is located at $z \sim 200-300 \mathrm{pc}$ above the Galactic midplane.

substantial atomic envelopes and show no evidence for hidden 'dark' material (either optically thick $\mathrm{H}$ I or CO-dark $\mathrm{H}_{2}$ ), implying that insufficient material exists to shield $\mathrm{CO}$ against photodissociation. Some also show shock-disrupted morphology, leading to the interpretation that these small clouds are pre-existing molecular gas currently undergoing dynamical disruption, gas stripping, and eventual dissociation due to their interaction with the shell. Survival lifetimes are roughly estimated as a few Myr, both from reference to the numerical results described in Section 4.1 and through estimates of the photodissociation timescale.

Conversely, CO clouds well embedded within the main atomic shell walls are excellent candidates for newly formed clouds. The feature labelled ' $b$ ' in the figure is the strongest example of such an object. This cloud shows evidence for a substantial dark component identified from $100 \mu \mathrm{m}$ infrared excess, which comprises over $50 \%$ of the total mass of the $\mathrm{H} \mathrm{I}$ - $\mathrm{CO}$ complex, and provides sufficient material to shield $\mathrm{CO}$ molecules against UV dissociation, demonstrating that the cloud can survive and continue to grow in its present form. The mean initial density required to sweep up the complex from the ambient medium is $\sim 1-10 \mathrm{~cm}^{-3}$ (depending on the assumed geometry), consistent with a realistic mixture of WNM and CNM. Comparing with the numerical simulations of Section 3.3, and assuming a flow speed equal to the present-day expansion velocity of $v_{\exp } \sim 10 \mathrm{~km} \mathrm{~s}^{-1}$, this implies that timescales of $<10^{7} \mathrm{yr}$ are needed to form significant quantities of $\mathrm{CO}-$ consistent with the estimated age of the shell. In addition, Wünsch et al. (2012) argue that the mass spectrum of molecular clumps in this region is consistent with the predictions of pressure-assisted gravitational instability in an expanding shell (Wünsch et al. 2010), providing further constraints on the physics of the swept-up ISM.

The net effect of the two shells on the volumes they occupy is estimated by comparing the molecular fraction, $f_{\mathrm{H}_{2}}=M_{\mathrm{H}_{2}} /\left(M_{\mathrm{HI}}+M_{\mathrm{H}_{2}}\right)$, in shell volumes-including the evacuated voids - to that in their local vicinities (essentially a proxy for the undisturbed medium). Since $f_{\mathrm{H}_{2}}$ varies with location in the Galactic disc, these 'background' regions are restricted carefully to include only emission that is genuinely local to the shells. The results of this analysis reveal that $f_{\mathrm{H}_{2}}$ in the supershell volumes is enhanced by a factor of $\sim 2-$ 3 with respect to their local surroundings, implying that as much as $50 \%-70 \%$ of the molecular matter in their walls may have been formed as a direct result of stellar feedback. At present, this analysis is restricted to two objects-both of which were selected in part because of their association with molecular clouds-and no strong conclusions can be drawn about the Milky Way as a whole. Nevertheless, this is a compelling proof of concept, and some of the first quantitative evidence of molecular cloud formation in shell walls. 


\subsubsection{Cold H I in shell walls}

The presence of cold atomic medium in supershell walls is important, since it is a necessary precursor to molecular gas. Heiles (1982) reports measurements of H I absorption spectra in the walls of several nearby supershells, taken towards background continuum sources. They find that $\mathrm{H} \mathrm{I}$ in the shells is in the cold, neutral regime, with excitation temperatures ranging from 35 to $200 \mathrm{~K}$-much colder than temperatures outside the shell walls.

Dawson et al. (2011b) fit multiple Gaussian profiles to H I emission in selected subsections the walls of the two shells discussed in Section 5.3.1, and use the measured line widths to put a strict upper limit on the kinetic temperature of the gas of $\sim 350 \mathrm{~K}$. Assuming that turbulence contributes approximately half of the observed line width (e.g. Heiles \& Troland 2003), an estimate of $T_{K} \sim 100 \mathrm{~K}$ is found for the fitted sections of shell wall. Together with an estimated density of $\sim 10 \mathrm{~cm}^{-3}$, this implies that the atomic shell walls are dominated by cold gas with parameters close to the canonical values for the CNM (see also McClure-Griffiths et al. 2003).

$\mathrm{H}$ I self-absorption (HISA) provides a better probe of the morphology and physical properties of cold atomic clouds, although the requirement of bright background emission means that HISA is typically only observable at low Galactic latitudes. Moss et al. (2012) report the discovery of the very large supershell GSH 006-15+7, whose lower regions are seen as a striking cone-like absorption feature against the Galactic plane. They estimate optical depths and kinetic temperatures of $\tau \sim 3$ and $T_{K} \sim 40 \mathrm{~K}$, respectively, again indicating the presence of copious quantities of cold, opaque atomic material. A similar and more extreme result is reported by Knee \& Brunt (2001), who present evidence for an extremely massive $\left(\sim 10^{7} \mathrm{M}_{\odot}\right)$, extremely cold $(\sim 10$ $20 \mathrm{~K})$ atomic arc believed to belong to the distant outer Galaxy shell GSH 139-03-69.

Although little work on the thermal state of the atomic medium in supershells has been carried out beyond these studies, they provide strong evidence that much of the ISM in supershells is in the form of cold atomic gas, consistent with them playing an important role in ISM cooling and cloud formation.

\subsubsection{High-altitude molecular material}

Another role of supershells may be in supporting a molecular 'thick disc' (Dame \& Thaddeus 1994; Malhotra 1994a) by elevating molecular material well above its normal scale height $\left(\sigma_{z} \sim 60 \mathrm{pc}\right.$ at the solar circle Malhotra 1994b). Several studies have explicitly noted high-altitude molecular gas associated with feedback superstructures. Megeath et al. (2003) report the association of the large, high-altitude $(z \sim 300 \mathrm{pc})$ star-forming molecular cloud complex NGC 281 with an expanding supershell. Both of the shells described in detail in Section 5.3.1 also contain moderately sized $\left(\sim 10^{3}-10^{4} M_{\odot}\right)$ molecular clouds at heights of up to $z \sim 450 \mathrm{pc}$ above the mid-

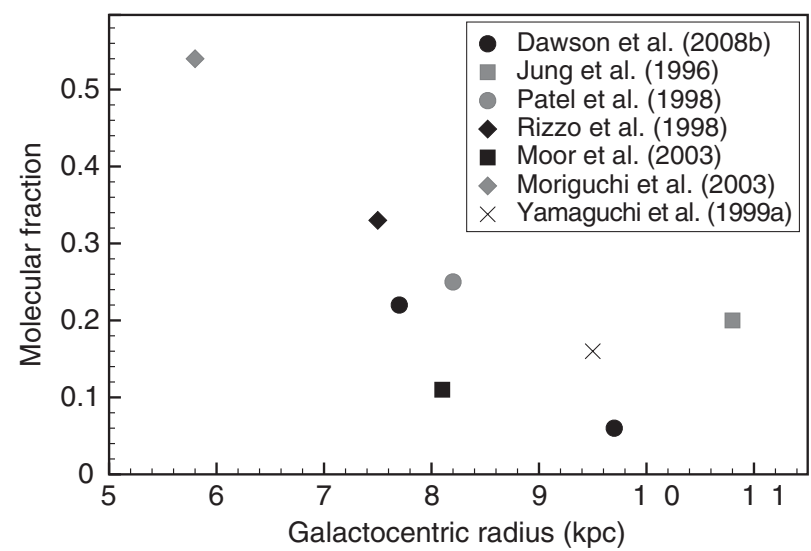

Figure 5. The relationship between Galactocentric radius and molecular fraction in Milky Way supershells. The molecular fraction, $f_{\mathrm{H}_{2}}=$ $M_{\mathrm{H}_{2}} /\left(M_{\mathrm{HI}}+M_{\mathrm{H}_{2}}\right)$ is calculated for all supershells in the literature for which estimates of both atomic and molecular mass are available for the full shell.

plane (Dawson et al. 2011b). This includes objects nominally identified both as pre-existing and newly formed clouds.

Matsunaga et al. (2001) also report the detection of eight supershell candidates initially identified from $\mathrm{CO}$ alone, mostly seen either as holes in the $\mathrm{CO}$ distribution or as strings of discrete molecular clouds that trace arc-like shapes above the plane. Six of these objects contain molecular gas at $z \gtrsim$ $150 \mathrm{pc}$. Moderately elevated clouds are also seen by Kim \& Koo (2000) and Moriguchi et al. (2002) in GW 46.4+5.5 and the M16/M17 supershell, respectively. Finally, Heiles et al. (1996) note the presence of 'high- $z$ CO' associated with two Galactic Worms, GW 30.5-2.5 and GW 49.1-1.4, though they do not quote $z$ itself. These results suggest that the molecular thick disc is supported at least in part by both the formation and the displacement of molecular gas due to discrete large-scale stellar feedback events.

\subsubsection{Remarks on molecular fraction and Galactocentric radius}

The picture of supershell-driven molecular cloud formation outlined in this review applies primarily to a regime in which the ambient medium contains a large atomic component. Mean densities must also be high enough that feedback flows are able to accumulate sufficient quantities of material for gas cooling and molecule formation. These conditions are applicable within a few kpc of the solar circle, but are unlikely to apply to the extreme inner or outer regions of the disc. The relevance of feedback-triggered molecular cloud formation is therefore likely to be a function of Galactocentric radius.

While quantitative measurements of molecular cloud formation in supershells are still too few to provide any observational insight into its variation with location, it is worth noting that available estimates of the $\mathrm{H}$ I and $\mathrm{H}_{2}$ masses of Galactic supershells do demonstrate the expected propensity for increasing molecular fraction with decreasing Galactocentric radius (see Figure 5). This also has implications for 
the initial detection and statistical study of populations of supershells, particularly when detections are based on a single tracer. The eight supershell candidates discovered in $\mathrm{CO}$ by Matsunaga et al. (2001) are all inner Galaxy objects, located between $R \sim 5.5$ and $7.5 \mathrm{kpc}$ (rescaled for $R_{0}=8.0$ $\mathrm{kpc}$ ). Conversely, $\mathrm{H}$ I -based detections may well be biassed against inner Galaxy shells, as decreasing ambient atomic gas fraction and increasing confusion renders coherent $\mathrm{H}$ I structures more difficult to detect.

\subsection{The Large Magellanic Cloud}

The LMC is the nearest star-forming galaxy to the Milky Way $(D \sim 50 \mathrm{kpc})$, and an excellent laboratory for studying the interaction between stars and the ISM. Unlike in the Milky Way, line-of-sight confusion is not a significant problem and the reliable identification of supershells is considerably simplified, enabling a more statistical approach to the question of molecular cloud formation. A large number of shells and supershells have been identified (e.g. Davies, Elliott, \& Meaburn 1976; Meaburn 1980; Chu \& Mac Low 1990; Kim et al. 1999), in a variety of observational tracers, and several studies have explicitly considered their relationship to the molecular ISM.

Yamaguchi et al. (2001b) make a statistical study of the effects of optically identified supergiant shells (SGSs, defined as those whose radii are larger than the disc scale height) on the formation of stars and molecular clouds. They find that both the number density and mass density of ${ }^{12} \mathrm{CO}(J=$ 1-0) molecular clouds is enhanced by a factor of 1.5-2 at the edges of SGSs, which they interpret as evidence of cloud formation. Book et al. (2009) examine a subset of this SGS population in detail and argue that both the compression of pre-existing dense clouds and the formation of new molecular gas are likely occurring in the supergiant shells LMC 1, 4, 5, and 6. The interacting shells LMC 4 and LMC 5 contain particularly convincing examples of both processes. A large, dense ridge of molecular material compressed between them forms a striking example of the classic picture of efficient cloud formation at the interface of two shells (e.g. Hartmann et al. 2001; Ntormousi et al. 2011), while a smaller cometary $\mathrm{CO}$ cloud with an $\mathrm{H}$ I tail exhibits the classic morphological signatures of the interaction of a shock front with pre-existing dense gas (see also Yamaguchi et al. 2001a). H I absorption spectra towards background continuum sources also suggest that efficient cooling has driven the production of large quantities of cold atomic gas in the walls of LMC 4 (Marx-Zimmer et al. 2000). This is consistent with the behaviour seen in Milky Way supershells.

Dawson et al. (2013) combine CO data with H I synthesis images (Kim et al. 2003) to make the first quantitative measurement of feedback-driven cloud formation in an entire galactic system. As described in Section 5.3.1 for Milky Way shells, their method compares the molecular fraction, $f_{\mathrm{H}_{2}}=M_{\mathrm{H}_{2}} /\left(M_{\mathrm{HI}}+M_{\mathrm{H}_{2}}\right)$, in SGS volumes with that else-

PASA, 30, e025 (2013)

doi:10.1017/pas.2013.002 where in the disc, in order to assess whether the passage of an SGS through the ISM is associated with a net increase in the production of molecular gas. Figure 6 shows an $\mathrm{H} \mathrm{I}$ and CO map of the LMC disc, overlaid with the outlines of the SGS complexes used in this analysis. They find that $f_{\mathrm{H}_{2}}$ in the aggregate volume occupied by all SGSs is identical to the rest of the LMC disc, suggesting that supergiant shells are not a dominant driver of molecular cloud formation on galactic scales. Indeed, the global structure of the LMC disc is found to be a better determinant of where the highest molecular fractions are found. However, the majority of objects ( $\sim 70 \%$ by mass) are more molecular than their local surroundings, implying that the presence of a supergiant shell does on average have a small positive effect on the molecular gas fraction. This analysis is used to place a lower limit on the total fraction of molecular cloud formation in the LMC driven by large-scale stellar feedback, which is estimated to be $\geq 4 \%-11 \%$ of the total molecular mass of the galaxy.

The importance of stellar feedback as a driver of molecular cloud formation is expected to be dependent on galaxy type. As a dwarf irregular, the LMC is expected to be more susceptible to feedback-triggered molecular cloud formation than early-type spirals such as the Milky Way (see also Section 3.2; Elmegreen et al. 2002). With low shear (Weidner, Bonnell, \& Zinnecker 2010), a large H I scale height (Brinks, Walter, \& Ott 2002) and weak spiral structure, shells in the LMC are able to grow larger before they are deformed, expand further before vertical blowout and depressurisation, and not suffer the spiral arm disruption that will affect their Milky Way counterparts. Similarly, the lack of strong spiral structure, combined with a relatively weak disc gravitational potential, suggests that galaxy-scale self-gravity and the accumulation of the ISM in spiral arms may play a less important role in dense gas formation than in grand-design spirals.

\section{SUMMARY, DISCUSSION, AND FUTURE DIRECTIONS}

The accumulation, compression, and cooling of the ISM in supershells formed by OB cluster feedback can drive the production of star-forming molecular clouds. Analytical and numerical models provide a strong theoretical basis for this process, and observations leave little doubt that molecular cloud formation via large-scale feedback is occurring in both the Milky Way and the LMC.

Theoretically, many details remain to be hammered out, including the ability of magnetic fields to prevent compressed material from reaching sufficient densities for molecule formation, whether multiple episodes of compression are necessary, and whether the initial ambient density must be higher than canonical WNM values in order for flow-driven cloud formation to occur. (e.g. Dobbs et al. 2012; Inoue \& Inutsuka 2012). Observationally, there are many convincing candidates for molecular clouds formed in supershell walls, and ample evidence that the atomic ISM in these shell walls 


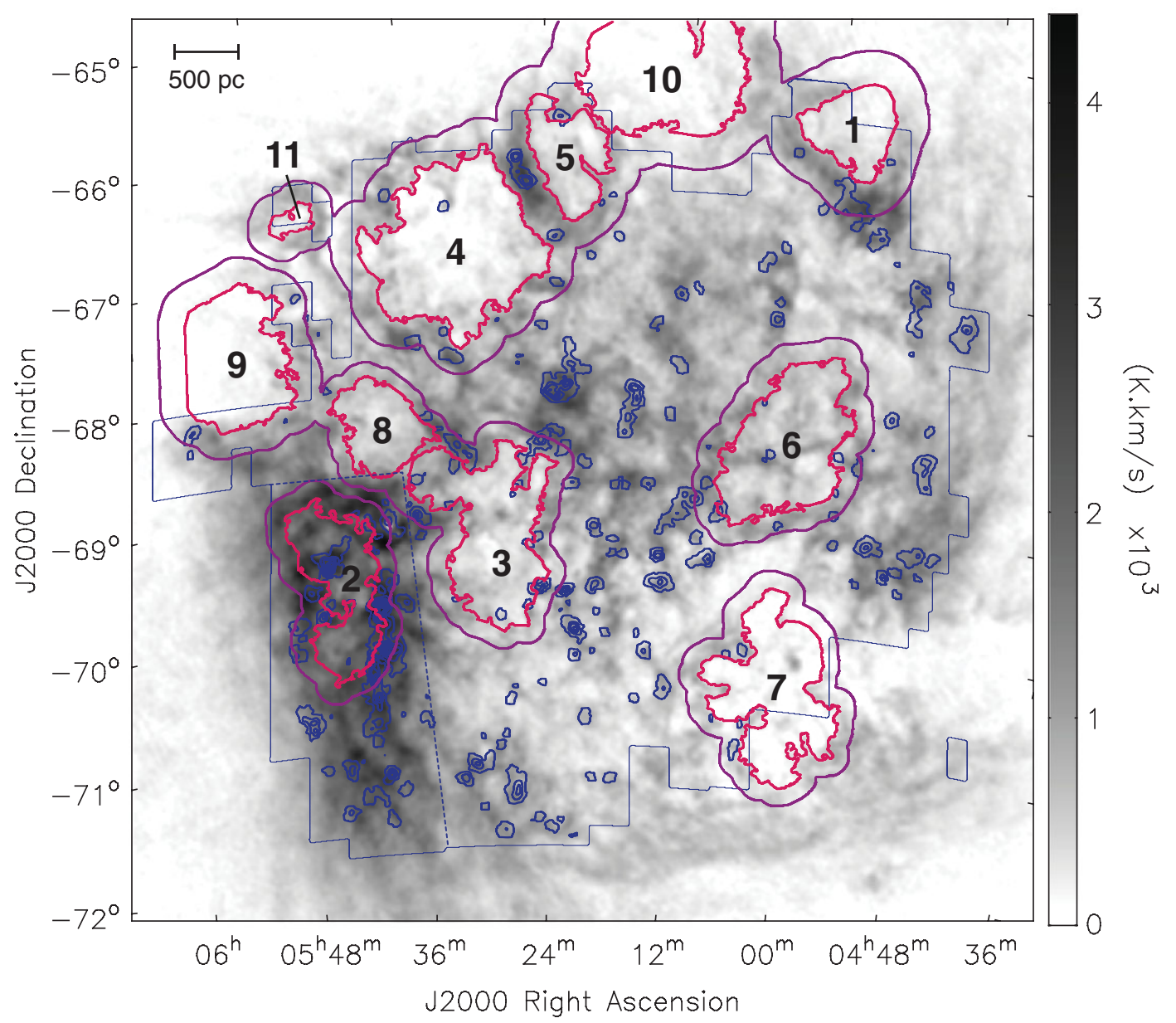

Figure 6. Supergiant shells and shell complexes overlaid on an integrated intensity map of the LMC (Reproduced by permission of the AAS from Dawson et al. 2013). The greyscale image is $\mathrm{H}$ I (Kim et al. 2003), and blue contours are ${ }^{12} \mathrm{CO}(J=1-0)$ (Fukui et al. 2008), processed as described in Dawson et al. (2013) and integrated over the full velocity range of the LMC disc. The solid blue line marks the boundary of the region observed in CO. Dark pink lines trace the inner rims of the shell complexes and purple lines mark their outer boundaries (delineating the outer edges of the dense shells). Dotted blue lines enclose the region known as the southeastern $\mathrm{H}$ I overdensity.

tends to be cold, demonstrating that the cooling of swept-up material occurs readily. Enhanced molecular gas fractions in the volumes occupied by two Galactic supershells have also provided one of the first quantitative measurements of feedback-driven cloud formation in the Milky Way (Dawson et al. 2011b). However, the question of how readily the process occurs remains open, as does its relative importance in the galactic context. The first direct measurement of the contribution of stellar feedback to molecular cloud formation on galactic scales has now been made in the LMC, where $\gtrsim 4 \%-11 \%$ of the total molecular gas content of the galaxy is estimated to have been formed as a direct result of large-scale feedback (Dawson et al. 2013). However, no such estimates exist for the Milky Way or other nearby galaxies.

Thinking globally, large-scale gravitational instabilities play a major role in the initial formation of dense gas in galaxy discs (see e.g. Elmegreen 2002; Mac Low \& Glover 2012, and references therein), and may be the primary driver of molecular gas formation in most systems. Spiral arms are also clearly an important means of accumulating material, although whether they drive a significant amount of molecular gas formation or whether their primary influence is to agglomerate existing GMCs into larger complexes is not yet clear (Koda et al. 2009). A picture is suggested in which large-scale feedback plays a secondary role-initiating further episodes of compression and additional cloud formation in an inhomogeneous medium that already contains significant amounts of dense structure. This picture is tentatively consistent with the results presented in this review. However, direct measurements of the contribution of stellar feedback to molecular cloud formation rates are strongly desirable.

Observational work that explicitly deals with feedbacktriggered cloud formation has generally taken a morphologybased approach, which relies on first identifying supershell structures and then examining the associated molecular ISM. This strategy requires that the neutral ISM be 
resolved to scales of $\sim 10-100 \mathrm{pc}$, which has historically been challenging for external galaxies, particularly in molecular line observations. With the advent of millimetre and submillimetre interferometers such as the Combined Array for Research in Millimeter Astronomy (CARMA) and ALMA, high-resolution, high-sensitivity observations of molecular tracers are now becoming possible in local group galaxies. This opens up the exciting possibility of extending the study of feedback-triggered molecular cloud formation to large samples of galaxies with a range of different properties. Such work will be essential in constructing a consistent model of the impact of stellar feedback on ISM evolution across a broad range of galaxy types.

Gaseous discs contain large amounts of stochastic structure, and the reliable identification of signatures of stellar feedback-particularly in the neutral ISM alone-is a source of uncertainty in observational studies. A preferable strategy would be a means of exploring feedback-triggered cloud formation that does not first rely on the morphological identification of shell-like structures. A promising approach is suggested by the work of Dobbs et al. (2012), who identify characteristic distributions in the eigenvalues of the local rate-of-strain tensor in model discs dominated by different astrophysical drivers of converging flows. This suggests the possibility of identifying the primary drivers of the flows that assemble dense clouds without recourse to morphological arguments, and hence quantifying the importance of stellar feedback in a robust and statistical sense. While it remains to be seen if this method can be usefully applied to real astronomical datasets (which cannot directly provide information on the 3D velocity field of the ISM), such direct marriages between simulations and observations are likely to provide powerful tools for the interpretation of future high-resolution data. This underscores the importance of using model galaxies - in which the input parameters are well constrained-to develop observational diagnostics for the ISM in real systems.

\section{ACKNOWLEDGMENTS}

My thanks to Eva Ntormousi and Alex Hill for their kind permission to make use of their figures in this paper.

\section{REFERENCES}

André, P., et al. 2010, A\&A, 518, L102

Audit, E., \& Hennebelle, P. 2005, A\&A, 433, 1

Bagetakos, I., Brinks, E., Walter, F., de Blok, W. J. G., Usero, A., Leroy, A. K., Rich, J. W., \& Kennicutt, R. C. Jr, 2011, AJ, 141, 23

Bally, J. 2001, ASPC, 231, 204

Banerjee, R., Vázquez-Semadeni, E., Hennebelle, P., \& Klessen, R. S. 2009, MNRAS, 398, 1082

Basu, S., Johnstone, D., \& Martin, P. G. 1999, ApJ, 516, 843

Bergin, E. A., Hartmann, L. W., Raymond, J. C., \& BallesterosParedes, J. 2004, ApJ, 612, 921
Book, L. G., Chu, Y.-H., Gruendl, R. A., \& Fukui, Y. 2009, AJ, 137, 3599

Boss, A. P. 1995, ApJ, 439, 224

Bournaud, F., Elmegreen, B. G., Teyssier, R., Block, D. L., \& Puerari, I. 2010, MNRAS, 409, 1088

Brand, P. W. J. L., \& Zealey, W. J. 1975, A\&A, 38, 363

Brinks, E., Walter, F., \& Ott, J. 2002, ASPC, 275, 57

Bruhweiler, F. C., Gull, T. R., Kafatos, M., \& Sofia, S. 1980, ApL, 238, 27

Carpenter, J. M., Heyer, M. H., \& Snell, R. L. 2000, ApJS, 130, 381

Chu, Y.-H., \& Mac Low, M.-M. 1990, ApJ, 365, 510

Clark, P. C., Glover, S. C. O., Klessen, R. S., \& Bonnell, I. A. 2012, MNRAS, 424, 2599

Comeron, F., \& Torra, J. 1992, A\&A, 261, 94

Comeron, F., \& Torra, J. 1994, A\&A, 281, 35

Cowie, L. L., \& McKee, C. F. 1977, ApJ, 211, 135

Dale, J. E., Bonnell, I. A., \& Whitworth, A. P. 2007, MNRAS, 375, 1291

Dale, J. E., Ercolano, B., \& Bonnell, I. A. 2012, MNRAS, 427, 2852

Dale, J. E., Wünsch, R., Whitworth, A., \& Palouš, J. 2009, MNRAS, 398, 1537

Dame, T. M., \& Thaddeus, P. 1994, ApL, 436, 173

Davies, R. D., Elliott, K. H., \& Meaburn, J. 1976, MmRAS, 81, 89

Dawson, J. R., Kawamura, A., Mizuno, N., Onishi, T., \& Fukui, Y. 2008a, PASJ, 60, 1297

Dawson, J. R., McClure-Griffiths, N. M., Dickey, J. M., \& Fukui, Y. 2011a, ApJ, 741, 85

Dawson, J. R., McClure-Griffiths, N. M., \& Fukui, Y. 2012, in EAS Publications Series, Vol. 56, ed. M. A. de Avillez, 155-158 (Les Ulis Cedex: EDP Sciences)

Dawson, J. R., Mizuno, N., Onishi, T., McClure-Griffiths, N. M., \& Fukui, Y. 2008b, MNRAS, 387, 31

Dawson, J. R., McClure-Griffiths, N. M., Kawamura, A., \& Mizuno, N. et al. 2011b, ApJ, 728, 127

Dawson, J. R., McClure-Griffiths, N. M., Wong, T., Dickey, J. M., Hughes, A., Fukui, Y., \& Kawamura, A. 2013, ApJ, 763, 56

de Avillez, M. A., \& Berry, D. L. 2001, MNRAS, 328, 708

de Avillez, M. A., \& Breitschwerdt, D. 2005, A\&A, 436, 585

de Geus, E. J. 1992, A\&A, 262, 258

de Geus, E. J., \& Burton, W. B. 1991, A\&A, 246, 559

Deharveng, L., et al. 2010, A\&A, 523, A6

Dennison, B., Topasna, G. A., \& Simonetti, J. H. 1997, ApL, 474, 31

Dickey, J. M. 2012, in EAS Publications Series, Vol. 56, ed. M. A. de Avillez, 371-379 (Les Ulis Cedex: EDP Sciences)

Digel, S. W., Lyder, D. A., Philbrick, A. J., Puche, D., \& Thaddeus, P. 1996, ApJ, 458, 561

Dobbs, C. L., \& Bonnell, I. A. 2008, MNRAS, 385, 1893

Dobbs, C. L., Bonnell, I. A., \& Pringle, J. E. 2006, MNRAS, 371, 1663

Dobbs, C. L., Burkert, A., \& Pringle, J. E. 2011, MNRAS, 417, 1318

Dobbs, C. L., Pringle, J. E., \& Burkert, A. 2012, MNRAS, 425, 2157

Draine, B. T., \& Bertoldi, F. 1996, ApJ, 468, 269

Efremov, Y. N., Ehlerová, S., \& Palouš, J. 1999, A\&A, 350, 457

Ehlerova, S., \& Palous, J. 1996, A\&A, 313, 478

Ehlerová, S., \& Palouš, J. 2002, MNRAS, 330, 1022

Ehlerová, S., \& Palouš, J. 2005, A\&A, 437, 101 
Ehlerova, S., Palous, J., Theis, C., \& Hensler, G. 1997, A\&A, 328, 121

Elmegreen, B. G. 2000, ApJ, 530, 277

Elmegreen, B. G. 2002, ApJ, 577, 206

Elmegreen, B. G., Palouš, J., \& Ehlerová, S. 2002, MNRAS, 334, 693

Federrath, C., \& Klessen, R. S. 2012, ApJ, 761, 156

Federrath, C., Roman-Duval, J., Klessen, R. S., Schmidt, W., \& Mac Low, M.-M. 2010, A\&A, 512, A81

Ferrière, K. M. 2001, RvMP, 73, 1031

Field, G. B., Goldsmith, D. W., \& Habing, H. J. 1969, ApL, 155, 149

Fragile, P. C., Murray, S. D., Anninos, P., \& van Breugel, W. 2004, ApJ, 604, 74

Franco, J., \& Cox, D. P. 1986, PASP, 98, 1076

Frisch, P. C., Redfield, S., \& Slavin, J. D. 2011, ARA\&A, 49, 237

Fukui, Y., Onishi, T., Abe, R., Kawamura, A., Tachihara, K., Yamaguchi, R., Mizuno, A., \& Ogawa, H. 1999, PASJ, 51, 751

Fukui, Y., et al. 2008, ApJS, 178, 56

Glover, S. C. O., \& Clark, P. C. 2012, MNRAS, 426, 377

Glover, S. C. O., \& Mac Low, M.-M. 2007, ApJ, 659, 1317

Glover, S. C. O., \& Mac Low, M.-M. 2011, MNRAS, 412, 337

Gregori, G., Miniati, F., Ryu, D., \& Jones, T. W. 1999, ApL, 527, 113

Grenier, I. A. 2004, ArXiv Astrophysics e-prints, arXiv:astro$\mathrm{ph} / 0409096$

Grenier, I. A., Casandjian, J., \& Terrier, R. 2005, Sci, 307, 1292

Grenier, I. A., Lebrun, F., Arnaud, M., Dame, T. M., \& Thaddeus, P. 1989, ApJ, 347, 231

Hartmann, L. 2003, ApJ, 585, 398

Hartmann, L., Ballesteros-Paredes, J., \& Bergin, E. A. 2001, ApJ, 562,852

Heiles, C. 1979, ApJ, 229, 533

Heiles, C. 1982, ApJ, 262, 135

Heiles, C. 1998, ApJ, 498, 689

Heiles, C., Haffner, L. M., \& Reynolds, R. J. 1999, ASPC, 168, 211

Heiles, C., Reach, W. T., \& Koo, B.-C. 1996, ApJ, 466, 191

Heiles, C., \& Troland, T. H. 2003, ApJ, 586, 1067

Heithausen, A., \& Thaddeus, P. 1990, ApL, 353, 49

Heitsch, F., \& Hartmann, L. 2008, ApJ, 689, 290

Heitsch, F., Hartmann, L. W., \& Burkert, A. 2008a, ApJ, 683, 786

Heitsch, F., Hartmann, L. W., Slyz, A. D., Devriendt, J. E. G., \& Burkert, A. 2008b, ApJ, 674, 316

Heitsch, F., Slyz, A. D., Devriendt, J. E. G., Hartmann, L. W., \& Burkert, A. 2006, ApJ, 648, 1052

Heitsch, F., Stone, J. M., \& Hartmann, L. W. 2009, ApJ, 695, 248

Hennebelle, P., Banerjee, R., Vázquez-Semadeni, E., Klessen, R. S., \& Audit, E. 2008, A\&A, 486, L43

Hennebelle, P., \& Pérault, M. 1999, A\&A, 351, 309

Heyer, M. H., \& Terebey, S. 1998, ApJ, 502, 265

Heyer, M. H., et al. 1996, ApL, 464, 175

Hill, A. S., Joung, M. R., Mac Low, M.-M., Benjamin, R. A., Haffner, L. M., Klingenberg, C., \& Waagan, K. 2012, ApJ, 750, 104

Hollenbach, D., \& Salpeter, E. E. 1971, ApJ, 163, 155

Hopkins, P. F., Quataert, E., \& Murray, N. 2012, MNRAS, 421, 3488

Hosokawa, T., \& Inutsuka, S.-i. 2006, ApJ, 646, 240

Inoue, T., \& Inutsuka, S. 2008, ApJ, 687, 303

Inoue, T., \& Inutsuka, S. 2009, ApJ, 704, 161
Inoue, T., \& Inutsuka, S.-i. 2012, ApJ, 759, 35

Joung, M. K. R., \& Mac Low, M.-M. 2006, ApJ, 653, 1266

Joung, M. R., Mac Low, M.-M., \& Bryan, G. L. 2009, ApJ, 704, 137

Jung, J. H., Koo, B.-C., \& Kang, Y.-H. 1996, AJ, 112, 1625

Kim, S., Dopita, M. A., Staveley-Smith, L., \& Bessell, M. S. 1999, AJ, 118, 2797

Kim, K.-T., \& Koo, B.-C. 2000, ApJ, 529, 229

Kim, W.-T., \& Ostriker, E. C. 2006, ApJ, 646, 213

Kim, W.-T., Ostriker, E. C., \& Stone, J. M. 2002, ApJ, 581, 1080

Kim, S., Staveley-Smith, L., Dopita, M. A., Sault, R. J., Freeman, K. C., Lee, Y., \& Chu, Y.-H. 2003, ApJS, 148, 473

Klein, R. I., McKee, C. F., \& Colella, P. 1994, ApJ, 420, 213

Knee, L. B. G., \& Brunt, C. M. 2001, Natur, 412, 308

Koda, J., et al. 2009, ApL, 700, 132

Kompaneets, A. S. 1960, SPhD, 5, 46

Koo, B., \& Heiles, C. 1988, in IAU Colloq. 101: Supernova Remnants and the Interstellar Medium, ed. R. S. Roger \& T. L. Landecker (Cambridge University Press, Cambridge, UK), 473

Kun, M. 1998, ApJS, 115, 59

Kundt, W., \& Mueller, P. 1987, Ap\&SS, 136, 281

Lallement, R., Welsh, B. Y., Vergely, J. L., Crifo, F., \& Sfeir, D. 2003, A\&A, 411, 447

Leão, M. R. M., de Gouveia Dal Pino, E. M., Falceta-Gonçalves, D., Melioli, C., \& Geraissate, F. G. 2009, MNRAS, 394, 157

Loeb, A., \& Perna, R. 1998, ApL, 503, 35

Lombardi, M., Lada, C. J., \& Alves, J. 2008, A\&A, 489, 143

Mac Low, M.-M., \& Glover, S. C. O. 2012, ApJ, 746, 135

Mac Low, M.-M., \& Klessen, R. S. 2004, RvMP, 76, 125

Mac Low, M., \& McCray, R. 1988, ApJ, 324, 776

Mac Low, M., McCray, R., \& Norman, M. L. 1989, ApJ, 337, 141

Maciejewski, W., Murphy, E. M., Lockman, F. J., \& Savage, B. D. 1996, ApJ, 469, 238

Malhotra, S. 1994a, ApJ, 437, 194

Malhotra, S. 1994b, ApJ, 433, 687

Marx-Zimmer, M., Herbstmeier, U., Dickey, J. M., Zimmer, F., Staveley-Smith, L., \& Mebold, U. 2000, A\&A, 354, 787

Mashchenko, S. Y., \& Silich, S. A. 1994, in Numerical Simulations in Astrophysics: Proceedings of the First Unam-Cray Supercomputing Workshop, ed. J. Franco, S. Lizano, L. Aguilar, \& E. Daltabuit (Cambridge University Press, Cambridge, UK), 202

Matsunaga, K., Mizuno, N., Moriguchi, Y., Onishi, T., Mizuno, A., \& Fukui, Y. 2001, PASJ, 53, 1003

McClure-Griffiths, N. M., Dickey, J. M., Gaensler, B. M., \& Green, A. J. 2002, ApJ, 578, 176

McClure-Griffiths, N. M., Dickey, J. M., Gaensler, B. M., \& Green, A. J. 2003, ApJ, 594, 833

McClure-Griffiths, N. M., Dickey, J. M., Gaensler, B. M., Green, A. J., Haynes, R. F., \& Wieringa, M. H. 2000, AJ, 119, 2828

McCray, R., \& Kafatos, M. 1987, ApJ, 317, 190

McCray, R., \& Snow, T. P. Jr, 1979, ARA\&A, 17, 213

McKee, C. F., \& Ostriker, E. C. 2007, ARA\&A, 45, 565

Meaburn, J. 1980, MNRAS, 192, 365

Megeath, S. T., Biller, B., Dame, T. M., Leass, E. L., Whitaker, R., \& Wilson, T. L. 2003, RMxAC, 15, 151

Melioli, C., de Gouveia Dal Pino, E. M., de La Reza, R., \& Raga, A. 2006, MNRAS, 373, 811

Mellema, G., Kurk, J. D., \& Röttgering, H. J. A. 2002, A\&A, 395, L13

Merín, B., et al. 2008, ApJS, 177, 551 
Meyerdierks, H., \& Heithausen, A. 1996, A\&A, 313, 929

Meyerdierks, H., Heithausen, A., \& Reif, K. 1991, A\&A, 245, 247

Miville-Deschênes, M.-A., et al. 2010, A\&A, 518, L104

Moór, A., \& Kiss, C. 2003, CoKon, 103, 149

Moriguchi, Y., Onishi, T., Mizuno, A., \& Fukui, Y. 2002, in The Proceedings of the IAU 8th Asian-Pacific Regional Meeting, Volume II, ed. S. Ikeuchi, J. Hearnshaw, \& T. Hanawa (Pedagogical University Press), 173

Moss, V. A., McClure-Griffiths, N. M., Braun, R., Hill, A. S., \& Madsen, G. J. 2012, MNRAS, 421, 3159

Nakamura, F., McKee, C. F., Klein, R. I., \& Fisher, R. T. 2006, ApJS, 164, 477

Norman, C. A., \& Ikeuchi, S. 1989, ApJ, 345, 372

Normandeau, M., Taylor, A. R., \& Dewdney, P. E. 1996, Natur, 380, 687

Ntormousi, E., Burkert, A., Fierlinger, K., \& Heitsch, F. 2011, ApJ, 731,13

Oey, M. S., \& Clarke, C. J. 1997, MNRAS, 289, 570

Oey, M. S., Clarke, C. J., \& Massey, P. 2001, in Dwarf Galaxies and Their Environment, ed. K. S. de Boer, R.-J. Dettmar, \& U. Klein (Shaker Verlag, Aachen, Germany), 181

Oey, M. S., \& García-Segura, G. 2004, ApJ, 613, 302

Oey, M. S., Watson, A. M., Kern, K., \& Walth, G. L. 2005, AJ, 129, 393

Olano, C. A. 1982, A\&A, 112, 195

Olano, C. A. 2001, AJ, 121, 295

Olano, C. A., Meschin, P. I., \& Niemela, V. S. 2006, MNRAS, 369, 867

Orlando, S., Bocchino, F., Reale, F., Peres, G., \& Pagano, P. 2008, ApJ, 678, 274

Orlando, S., Peres, G., Reale, F., Bocchino, F., Rosner, R., Plewa, T., \& Siegel, A. 2005, A\&A, 444, 505

Patel, N. A., Goldsmith, P. F., Heyer, M. H., Snell, R. L., \& Pratap, P. 1998, ApJ, 507, 241

Pittard, J. M. 2011, MNRAS, 411, L41

Pittard, J. M., Falle, S. A. E. G., Hartquist, T. W., \& Dyson, J. E. 2009, MNRAS, 394, 1351

Pittard, J. M., Hartquist, T. W., \& Falle, S. A. E. G. 2010, MNRAS, 405,821

Poppel, W. 1997, FCPh, 18, 1

Preibisch, T., \& Zinnecker, H. 2007, IAUS, 237, 270

Reach, W. T., Koo, B.-C., \& Heiles, C. 1994, ApJ, 429, 672

Reynolds, R. J., Sterling, N. C., Haffner, L. M., \& Tufte, S. L. 2001, ApL, 548, 221

Rizzo, J. R., \& Arnal, E. M. 1998, A\&A, 332, 1025

Sedov, L. I. 1959, Similarity and Dimensional Methods in Mechanics (New York: Academic Press)

Shetty, R., \& Ostriker, E. C. 2008, ApJ, 684, 978

Shin, M.-S., Stone, J. M., \& Snyder, G. F. 2008, ApJ, 680, 336

Tachihara, K., Toyoda, S., Onishi, T., Mizuno, A., Fukui, Y., \& Neuhäuser, R. 2001, PASJ, 53, 1081
Tasker, E. J. 2011, ApJ, 730, 11

Tasker, E. J., \& Tan, J. C. 2009, ApJ, 700, 358

Taylor, A. R., Irwin, J. A., Matthews, H. E., \& Heyer, M. H. 1999, ApJ, 513, 339

Taylor, D. K., Dickman, R. L., \& Scoville, N. Z. 1987, ApJ, 315, 104

Tenorio-Tagle, G. 1981, A\&A, 94, 338

Tenorio-Tagle, G., \& Bodenheimer, P. 1988, ARA\&A, 26, 145

Tenorio-Tagle, G., \& Palous, J. 1987, A\&A, 186, 287

Tenorio-Tagle, G., Rozyczka, M., \& Bodenheimer, P. 1990, A\&A, 237, 207

Tielens, A. G. G. M., \& Hollenbach, D. 1985, ApJ, 291, 722

Tomisaka, K. 1992, PASJ, 44, 177

Tomisaka, K. 1998, MNRAS, 298, 797

Tomisaka, K., Habe, A., \& Ikeuchi, S. 1981, Ap\&SS, 78, 273

Tothill, N. F. H., et al. 2009, ApJS, 185, 98

van Dishoeck, E. F., \& Black, J. H. 1988, ApJ, 334, 771

Vanhala, H. A. T., \& Cameron, A. G. W. 1998, ApJ, 508, 291

Vázquez-Semadeni, E. 2010, ASPC, 438, 83

Vázquez-Semadeni, E., Gómez, G. C., Jappsen, A. K., BallesterosParedes, J., González, R. F., \& Klessen, R. S. 2007, ApJ, 657, 870

Vázquez-Semadeni, E., Ryu, D., Passot, T., González, R. F., \& Gazol, A. 2006, ApJ, 643, 245

Wada, K., \& Norman, C. A. 2001, ApJ, 547, 172

Wada, K., Spaans, M., \& Kim, S. 2000, ApJ, 540, 797

Walch, S. K., Whitworth, A. P., Bisbas, T., Wünsch, R., \& Hubber, D. 2012, MNRAS, 427, 625

Walder, R., \& Folini, D. 1996, A\&A, 315, 265

Warren, S. R., et al. 2011, ApJ, 738, 10

Weaver, R., McCray, R., Castor, J., Shapiro, P., \& Moore, R. 1977, ApJ, 218, 377

Weidner, C., Bonnell, I. A., \& Zinnecker, H. 2010, ApJ, 724, 1503

Weisz, D. R., Skillman, E. D., Cannon, J. M., Dolphin, A. E., Kennicutt, R. C. Jr, Lee, J., \& Walter, F. 2009, ApJ, 704, 1538

Wolfire, M. G., Hollenbach, D., \& McKee, C. F. 2010, ApJ, 716, 1191

Wolfire, M. G., Hollenbach, D., McKee, C. F., Tielens, A. G. G. M., \& Bakes, E. L. O. 1995, ApJ, 443, 152

Wünsch, R., Dale, J. E., Palouš, J., \& Whitworth, A. P. 2010, MNRAS, 407, 1963

Wünsch, R., Jáchym, P., Sidorin, V., Ehlerová, S., Palouš, J., Dale, J., Dawson, J. R., \& Fukui, Y. 2012, A\&A, 539, A116

Yamaguchi, N., Mizuno, N., Moriguchi, Y., Yonekura, Y., Mizuno, A., \& Fukui, Y. 1999a, PASJ, 51, 765

Yamaguchi, R., Mizuno, N., Onishi, T., Mizuno, A., \& Fukui, Y. 2001a, ApL, 553, 185

Yamaguchi, R., Mizuno, N., Onishi, T., Mizuno, A., \& Fukui, Y. 2001b, PASJ, 53, 959

Yamaguchi, R., Saito, H., Mizuno, N., Mine, Y., Mizuno, A., Ogawa, H., \& Fukui, Y. 1999b, PASJ, 51, 791 